\title{
Numerical Treatment of Eigenvalue Problems for Differential Equations with Discontinuous Coefficients*
}

\author{
By I. Babuska and J. E. Osborn
}

\begin{abstract}
The eigenvalues of a second order differential equation are approximated by "factoring" the second order equations into a first order system and then applying the Ritz-Galerkin method to this system. Convergence results and error estimates are derived. These error estimates are based on the application of Sobolev spaces with variable order.
\end{abstract}

\section{Chapter 1. InTRODUCtion}

During the last several years the theory of finite element methods has been extensively developed. The main thrust has been on the development of approximation methods and associated asymptotic error estimates which are based on a variational formulation of the problem to be solved. This work has mainly been based on the assumption of sufficient regularity of the solution, and the application of Sobolev spaces with constant (fractional) order. It has been shown that many methods can be obtained through the use of different variational formulations.

In particular, it has proved useful to "factor" a second order equation into a system of first order equations, to consider a variational formulation of this system, and then to apply the Ritz-Galerkin method associated with this variational formulation. The so-called mixed method is an example of such a method. Consider, for example, the equation

$$
-\operatorname{div}(A \operatorname{grad} u)+u=f
$$

This equation can be written as a system as follows:

$$
\begin{aligned}
A \operatorname{grad} u & =\sigma, \\
-\operatorname{div} \sigma+u & =f .
\end{aligned}
$$

If we now consider a Ritz-Galerkin approximation method based on a variational formulation of (1.1) and (1.2), we have the mixed method; see, e.g., Herrmann [10], Oden and Reddy [21], Babuska, Oden and Lee [3], and Raviart and Thomas [24]. For additional references see also [20, p. 290].

This general method (i.e., factoring a second order equation into a system of first order equations and then basing a Ritz-Galerkin approximation on a variational formulation

Received April 28, 1977; revised March 29, 1978.

AMS (MOS) subject classifications (1970). Primary 35P15, 65N15, 65N25, 65N30.

* The work of the first author was partially supported by the U. S. Energy Research and Development Administration under Contract E(40-1)3443 and that of the second author by NSF Grants GP35666 and MCS76-06963. 
of the resulting first order system) can be applied to both source problems and eigenvalue problems. Nemat-Nasser [12], [13], [14] has observed that this method is effective for the approximation of eigenvalues of problems with rough coefficients (coefficients possessing only a low degree of differentiability). These papers (and other related papers of Nemat-Nasser and co-authors [15], [16]) describe and elaborate the method and present some very interesting computational results. In [14], in which the approximations are based on trigonometric polynomials, a posteriori error bounds are proved (under certain assumptions on the spectrum) and the effectivity of the method is demonstrated by means of numerical examples. A priori convergence and rate of convergence is not established in these papers. Recently Conuto [6] has studied eigenvalue approximation in an abstract context and applied his results to problems with smooth coefficients.

In this paper we will study the simplest model problem, namely the one dimensional eigenvalue problem

$$
\begin{aligned}
-\left(u^{\prime} / \tau(x)\right)^{\prime}+\xi(x) u & =\lambda \rho(x) u, \quad 0<x<2 \pi, \\
u(0) & =u(2 \pi), \\
\left(u^{\prime} / \tau\right)(0) & =\left(u^{\prime} / \tau\right)(2 \pi),
\end{aligned}
$$

where $\tau, \xi$ and $\rho$ are real, measurable, $2 \pi$-periodic functions satisfying

$$
0<1 / M \leqslant \tau(x), \xi(x), \rho(x) \leqslant M .
$$

$\tau, \xi$ and $\rho$ could be, for example, step functions. Letting $\sigma=u^{\prime} / \tau$, we can write (1.3)(1.5) as the system

$$
\begin{gathered}
u^{\prime}=\tau \sigma, \\
-\sigma^{\prime}+\xi u=\lambda \rho u, \\
u(0)=u(2 \pi), \quad \sigma(0)=\sigma(2 \pi) .
\end{gathered}
$$

Taking the inner product of (1.6) with $s$ and (1.7) with $v$ and adding the results, we obtain

$$
\int_{0}^{2 \pi}\left(u^{\prime} \bar{s}-\tau \sigma \bar{s}-\sigma^{\prime} \bar{v}+\xi u \bar{v}\right) d x=\lambda \int_{0}^{2 \pi} \rho u \bar{v} d x .
$$

Here we seek a nonzero eigenfunction $(u, \sigma)$ such that $(1.8)$ holds for all $(v, s)$. We now obtain an approximation method by considering the Ritz-Galerkin method associated with the variational formulation (1.8), i.e., we seek approximations by restricting the variational equation (1.8) to an appropriately chosen finite dimensional space $S_{1}^{h} \times$ $S_{2}^{h}$. We will refer to the resulting method as the mixed method for eigenvalue calculation. In this paper we prove that the mixed method leads to convergent eigenvalue approximations and establish rate of convergence estimates. We are particularly interested in the case in which the coefficients $\tau, \xi$ and $\rho$ are rough.

It is well known that the rate of convergence for the standard Ritz method for the calculation of eigenvalues depends, in general, on the regularity (degree of differentiability) of the exact eigenfunction $u$. For the mixed method we will show that 


$$
\left|\lambda-\lambda_{n}\right| \leqslant C h^{-\delta}\left(\inf _{\chi \in S_{1}^{h}}\|u-\chi\|_{\alpha}^{2}+\inf _{\eta \in S_{2}^{h}}\|\sigma-\eta\|_{1-\alpha}^{2}\right),
$$

where $\|\cdot\|_{\alpha}$ denotes the fractional Sobolev norm, $\alpha$ is any index between 0 and 1 , and $\delta$ is a constant depending on $\alpha$ and the family $\left\{S_{1}^{h} \times S_{2}^{h}\right\}_{h}$. Thus, for the mixed method the rate of convergence depends on the regularity of $u$ and $\sigma$, and we can take advantage of the relation between the regularity of $u$ and that of $\sigma$, i.e., we can optimize the rate of convergence obtained in the above estimate by adjusting the index $\alpha$ to fit the regularity of $u$ and $\sigma$. We note that the choice of $\alpha$ does not affect the approximation method, but only affects the rate of convergence that can be established (by our results). This leads to a rate of convergence for the mixed method that is higher than that for the standard Ritz method.

The regularity of $u$ and $\sigma$ depend in turn on the regularity of the coefficients $\tau, \xi$ and $\rho$. It can thus easily happen that $u$ and $\sigma$ have different regularity in different places (i.e., in different parts of the interval $[0,2 \pi]$ ). We show that such local effects can be accounted for by taking the index $\alpha$ to be variable (i.e., a function of $x$ ). This leads naturally to the introduction of Sobolev space with variable order-in contrast to the unusual ones with constant (fractional) order-and to the development of eigenvalue approximation results based on these spaces. Thus, for problems with variable coefficients, in particular rough coefficients, the rate of convergence can be further improved by choosing $\alpha$ variable in an appropriate way. We note that this further improvement is possible when the approximation is based on finite elements but not when it is based on trigonometric polynomials. This difference is due to the local approximability properties of finite elements, in contrast to the nonlocal approximability properties of trigonometric polynomials.

The theory of Sobolev spaces with variable order has been developed and used in other connections in [25], [26], [27], [28], [29], [30]. In the Appendix (which appears in the microfiche section of this issue) we give a self-contained treatment of the part of this theory needed in our applications; the development in the Appendix is based on [26], [27]. Chapter 2 contains a summary of this material. In Chapter 3 we analyze the Ritz-Galerkin method for the approximation of the eigenvalues of (1.3)(1.5) which is based on (1.8). Asymptotic error estimates are established. In Sections 3.4 and 3.5 the results are summarized and compared with the results which could be obtained using Sobolev spaces with constant order. Computational examples and their analysis in terms of the theory developed here will be presented in a forthcoming paper.

The authors would like to thank their colleague Professor Raymond Johnson for helpful discussions on several topics in this paper.

\section{Chapter 2. Sobolev SPaces With VARIAble ORDER}

In this chapter we define the Sobolev spaces with variable order and state the results concerning them which we will use in the remainder of the paper. We give a selfcontained treatment of this material in the Appendix (see the microfiche section).

We denote by $E$ the set of all infinitely differentiable, complex valued, $2 \pi$-periodic functions and by $E_{R}$ the subset of functions in $E$ which are real valued. For 
$\alpha=\alpha(x) \in E_{R}$ we write

$$
\alpha_{+}=\max _{0 \leqslant x \leqslant 2 \pi} \alpha(x), \quad \alpha_{-}=\min _{0 \leqslant x \leqslant 2 \pi} \alpha(x) .
$$

For any real number $r$ we denote by $H^{r}$ the one dimensional, $2 \pi$-periodic Sobolev space of fractional order $r ; H^{r}$ is the completion of $E$ with respect to norm

$$
\|u\|_{r}=\left\{\sum_{k=-\infty}^{+\infty}\left|a_{k}(u)\right|^{2}(1+|k|)^{2 r}\right\}^{1 / 2},
$$

where $a_{k}(u)$ is the $k$ th Fourier coefficient of $u$. Defining $\Lambda^{r}: E \rightarrow E$ by

$$
\left(\Lambda^{r} u\right)(x)=\sum_{k} a_{k}(u)(1+|k|)^{r} e^{i k x},
$$

we have $\|u\|_{r}=\left\|\Lambda^{r} u\right\|_{0}$. If $-L \leqslant r$, it is easily seen that $\|u\|_{r}$ is equivalent to the norm

$$
\left(\left\|\Lambda^{r} u\right\|_{0}^{2}+\|u\|_{-L}^{2}\right)^{1 / 2}
$$

This expression will now be the starting point for the definition of spaces with variable order. For $\alpha=\alpha(x) \in E_{R}$ and $u \in E$ we define $\Lambda^{\alpha}=\Lambda^{\alpha(x)}: E \rightarrow E$ by

$$
\left(\Lambda^{\alpha} u\right)(x)=\sum_{k} a_{k}(u)(1+|k|)^{\alpha(x)} e^{i k x} \text {. }
$$

For $s$ real and $-L \leqslant \alpha_{-}+s$ we define

$$
\|u\|_{\alpha(x), s, L}=\left(\left\|\Lambda^{\alpha} u\right\|_{s}^{2}+\|u\|_{-L}^{2}\right)^{1 / 2} \text {, }
$$

and then define $H^{\alpha(x), s, L}$ to be the completion of $E$ with respect to $\|\cdot\|_{\alpha(x), s, L}$. At several points in the development of this theory, $L$ is required to be large, with the requirement on the size of $L$ depending on $\alpha(x)$ and $s$. Throughout the paper we assume $L$ has a fixed large positive value.

We now state four theorems which give the basic properties of the operator $\Lambda^{\alpha(x)}$.

THEOREM 1. Let $\alpha(x) \in E_{R}$ and $s$ be real. Then, for each $\epsilon>0$ there is a constant $C(\epsilon, \alpha(x), s)$ such that

for all $u \in E$.

$$
\left\|\Lambda^{\alpha(x)} u\right\|_{s} \leqslant C(\epsilon, \alpha(x), s)\|u\|_{\alpha_{+}+s+\epsilon}
$$

Theorem 2. Let $\alpha(x), \beta(x) \in E_{R}$ and $s$ be real. Then

where

$$
\Lambda^{\alpha(x)} \Lambda^{\beta(x)} u=\Lambda^{\alpha(x)+\beta(x)} u+w,
$$

for any $u \in E$.

$$
\|w\|_{s} \leqslant C(\epsilon, \alpha(x), \beta(x), s)\|u\|_{\alpha_{+}+\beta_{+}+s+\epsilon-1}
$$

THEOREM 3. Let $\alpha(x) \in E_{R}$ with $\alpha_{+}-\alpha_{-}<1$ and suppose $\alpha_{0}<\alpha_{-}$and $s$ is real. Then, for any $L$ there is a constant $C\left(\alpha(x), \alpha_{0}, s, L\right)$ such that

for all $u \in E$.

$$
\|u\|_{\alpha_{0}+s} \leqslant C\left(\alpha(x), \alpha_{0}, s, L\right)\left[\left\|\Lambda^{\alpha(x)} u\right\|_{s}+\|u\|_{-L}\right]
$$

TheOREM 4. Let $\alpha(x) \in E_{R}$ and $s$ be real. Then

where

$$
\Lambda^{\alpha(x)} u^{\prime}=\left(\Lambda^{\alpha(x)} u\right)^{\prime}+w \quad\left(^{\prime}=d / d x\right)
$$




$$
\|w\|_{s} \leqslant C(\epsilon, \alpha(x), s)\|u\|_{\alpha_{+}+s+\epsilon}
$$

for any $u \in E$.

We next present some notation which is used in the remaining theorems. These theorems contain the basic properties of the norms $\|\cdot\|_{\alpha(x), s, L}$ and the spaces $H^{\alpha(x), s, L}$. We now suppose $\alpha \in E_{R}$ with $\alpha_{+}-\alpha_{-}<1$.

Let $\chi$ be an infinitely differentiable function satisfying

$$
\begin{aligned}
& 0 \leqslant \chi(x) \leqslant 1, \quad \chi(-x)=\chi(x), \\
& \chi(x)=1 \quad \text { for }|x| \leqslant 1 / 2, \\
& \operatorname{supp} \chi \subset(-1,1) \text { and } \\
& \chi(x)+\chi(x-3 / 2)=1 \text { for } 1 / 2<x<1 .
\end{aligned}
$$

For any $0<\delta<\pi$ denote by $\chi_{\delta}$ that function in $E_{R}$ defined by

Then $\chi_{\delta}$ satisfies

$$
\chi_{\delta}(x)=\chi(x / \delta), \quad|x| \leqslant \pi .
$$

$$
\begin{aligned}
& 0 \leqslant \chi_{\delta}(x) \leqslant 1, \quad \chi_{\delta}(-x)=\chi_{\delta}(x), \\
& \chi_{\delta}(x)=1 \text { for }|x| \leqslant \delta / 2, \\
& \left(\operatorname{supp} \chi_{\delta}\right) \cap[-\pi, \pi] \subset(-\delta, \delta) \text { and } \\
& \chi_{\delta}+\chi_{\delta}(x-3 \delta / 2)=1 \text { for } \delta / 2<x<\delta .
\end{aligned}
$$

For $M=3,4, \ldots$ let $\theta=4 \pi / 3 M$ and define

$$
\chi_{\theta, j}(x)=\chi_{\theta}(x-3 j \theta / 2), \quad j=1,2, \ldots, M .
$$

It is readily seen that

$$
\sum_{j=1}^{M} \chi_{\theta, j}=1
$$

For any $u \in E$ we define

Then we have

$$
u_{\theta, j}=\chi_{\theta, j} u, \quad j=1, \ldots, M .
$$

$$
u=\sum_{j=1}^{M} u_{\theta, j}
$$

For $j=1, \ldots, M$ we consider the intervals $I_{\theta, j}=[3 j \theta / 2-4 \theta, 3 j \theta / 2+4 \theta]$ and suppose we are given real numbers $p_{\theta, j}^{-}<p_{\theta, j}^{+}, j=1, \ldots, M$, such that

$$
\max _{1 \leqslant j \leqslant M} p_{\theta, j}^{+}-\min _{1 \leqslant j \leqslant M} p_{\theta, j}^{-}<1 \text {. }
$$

We let $\vec{p}_{\theta, j}=\left(p_{\theta, j}^{-}, p_{\theta, j}^{+}\right)$and $\vec{p}_{\theta}=\left\{\vec{p}_{\theta, m}\right\}_{j=1}^{M}$. For $\alpha \in E_{R}$ with $\alpha_{+}-\alpha_{-}<1$, and $\theta(=4 \pi / 3 M)$ and $\vec{p}_{\theta}$ given, we write $\alpha(x) \sim \vec{p}_{\theta}$ if

$$
p_{\theta, j}^{-}<\alpha(x)<p_{\theta, j}^{+} \text {for } x \in I_{\theta, j}, j=1, \ldots, M \text {. }
$$

Theorem 5. If $\alpha \in E_{R}$ satisfies $\alpha_{+}-\alpha_{-}<1$, s is real and $(\alpha(x)+s) \sim \vec{p}_{\theta}$, then 


$$
C_{1}\left\{\sum_{j=1}^{M}\left\|u_{\theta, j}\right\|_{p_{\theta, j}}+\|u\|_{-L}\right\} \leqslant\|u\|_{\alpha, s, L} \leqslant C_{2}\left\{\sum_{j=1}^{M}\left\|u_{\theta, j}\right\|_{p_{\theta, j}^{+}}+\|u\|_{-L}\right\}
$$

for all $u \in E$. The constants $C_{1}, C_{2}$ depend on $\vec{p}_{\theta}, \alpha, s$, and $L$, but are independent of $u$.

THEOREM 6. Suppose $\alpha_{1}, \alpha_{2} \in E_{R}$ satisfy $\alpha_{j,+}-\alpha_{j,-}<1, j=1,2, s_{1}, s_{2}$ are real and

for all $x$. Then

$$
\alpha_{1}(x)+s_{1}<\alpha_{2}(x)+s_{2}
$$

$$
\|u\|_{\alpha_{1}, s_{1}, L} \leqslant C\|u\|_{\alpha_{2}, s_{2}, L}
$$

for all $u \in E$. Thus, $H^{\alpha_{2}, s_{2}, L} \subset H^{\alpha_{1}, s_{1}, L}$ with a continuous imbedding.

TheOREM 7. Suppose $\alpha_{1}, \alpha_{2} \in E_{R}$ satisfy $\alpha_{j,+}-\alpha_{j,-}<1, j=1,2$, and

$$
\alpha_{1}(x)<\alpha_{2}(x) \text { for all } x \text {. }
$$

Then the imbedding of $H^{\alpha_{2}, s, L}$ in $H^{\alpha_{1}, s, L}$ is compact.

THEOREM 8. Suppose $\alpha \in E_{R}$ satisfies $\alpha_{+}-\alpha_{-}<1$. Then

$$
C_{1}\|u\|_{\alpha, s, L} \leqslant \sup _{v \in E} \frac{\left|\int_{0}^{2 \pi} u \bar{v} d x\right|}{\|v\|_{-\alpha,-s, L}} \leqslant C_{2}\|u\|_{\alpha, s, L}
$$

for all $u \in E$, where $C_{1}$ and $C_{2}$ are positive constants.

THEOREM 9. Suppose $\alpha$ and $\beta$ are constants satisfying $|\alpha| \leqslant \beta, 1 / 2<\beta$. Then, there is a constant $C$ such that

$$
\|u v\|_{\alpha} \leqslant C\|u\|_{\alpha}\|v\|_{\beta}
$$

for all $u, v \in E$ (see also Strichartz [23]).

THEOREM 10. Suppose $\alpha(x), \beta(x), \gamma(x) \in E_{R}$ satisfy $\max (\alpha(x), \beta(x))>1 / 2$, $-\max (\alpha(x), \beta(x))<\gamma(x)<\min (\alpha(x), \beta(x))$, and $\alpha_{+}-\alpha_{-}<1, \beta_{+}-\beta_{-}<1, \gamma_{+}-$ $\gamma_{-}<1$. Then, there is a constant $C$ such that

for all $u, v \in E$.

$$
\|u v\|_{\gamma(x), 0, L} \leqslant C\|u\|_{\alpha(x), 0, L}\|v\|_{\beta(x), 0, L}
$$

Our final theorem is useful in determining which of the spaces $H^{\alpha(x), 0, L}$ a specific function lies in.

THEOREM 11. Suppose $u=\Sigma_{j=1}^{l} u_{j}$ where $u_{j} \in H^{p_{j}}$ with $p_{j} \geqslant-L$ and supp $u_{j}$ $\subset\left[\alpha_{j}, \beta_{j}\right]^{\prime}$ with $\beta_{j}-\alpha_{j}<\pi / 4$, where $\left[\alpha_{j}, \beta_{j}\right]^{\prime}$ denotes the union of $\left[\alpha_{j}, \beta_{j}\right]$ and all of its translates by $2 k \pi$. Let $\delta>(3 / 2) \max \left(\beta_{j}-\alpha_{j}\right)$ and suppose $s$ is real, $\alpha \in E_{R}$ satisfies $\alpha_{+}-\alpha_{-}<1$, and that

$$
-L<\alpha(x)+s<p_{j}, \text { for } x \in\left[\alpha_{j}-\delta, \beta_{j}+\delta\right], j=1, \ldots, l .
$$

Then $u \in H^{\alpha(x), s, L}$.

We mention briefly an application of this result. Let $u(x)$ be a $2 \pi$-periodic step function defined by

$$
u(x)= \begin{cases}k_{1}, & 0 \leqslant x \leqslant x_{1} \text { or } x_{2} \leqslant x<2 \pi, \\ k_{2}, & x_{1}<x<x_{2}\end{cases}
$$


where $0<x_{1}<x_{2}<2 \pi$. Let $\alpha(x) \in E_{R}$ satisfy $0<\alpha(x), \alpha_{+}-\alpha_{-}<1$, and $\alpha\left(x_{1}\right)$, $\alpha\left(x_{2}\right)<1 / 2$. Then, using Theorem 11 we can show that $u \in H^{\alpha(x), 0, L}$.

\section{Chapter 3. The eigenvalue Problem}

3.1. Introduction and Formulation of the Problem. We shall study the eigenvalue problem

$$
\begin{aligned}
-\left(u^{\prime} / \tau(x)\right)^{\prime}+\xi(x) u & =\lambda \rho(x) u, \quad 0<x<2 \pi, \\
u(0) & =u(2 \pi), \\
\left(u^{\prime} / \tau\right)(0) & =\left(u^{\prime} / \tau\right)(2 \pi) .
\end{aligned}
$$

We assume that $\tau, \xi$ and $\rho$ are real, measurable, $2 \pi$-periodic functions satisfying $0<$ $1 / M \leqslant \tau(x), \xi(x), \rho(x) \leqslant M$. This is a selfadjoint, positive definite eigenvalue problem with eigenvalues $\lambda_{j}$ and corresponding eigenfunctions $u_{j}$ :

$$
\begin{gathered}
0<\lambda_{1} \leqslant \lambda_{2} \leqslant \ldots \uparrow \infty \\
u_{1}, u_{2}, \ldots, \quad \int_{0}^{2 \pi} \rho u_{i} u_{j} d x=\delta_{i j} .
\end{gathered}
$$

The eigenvalue problem (1.1)-(1.3) has the following standard variational formulation. $\lambda$ is an eigenvalue if there is a corresponding nonzero eigenfunction $u \in H^{1}\left(H^{1}\right.$ is the $2 \pi$-periodic Sobolev space of order 1 ) such that

$$
\int_{0}^{2 \pi} \frac{1}{\tau} u^{\prime} \bar{\zeta}^{\prime} d x+\int_{0}^{2 \pi} \xi u \bar{\zeta} d x=\lambda \int_{0}^{2 \pi} \rho u \bar{\zeta} d x, \text { for all } \phi \in H^{1} .
$$

(1.1) $-(1.3)$ and (1.4) are equivalent in the sense that (1.4) is satisfied if and only if $u$ and $u^{\prime} / \tau \in H^{1}$ and (1.1) is satisfied almost everywhere. Thus, the eigenfunctions $u_{j}$ of (1.1)-(1.3) are in $H^{1}$; but it can be shown that there exist $\tau, \xi$ and $\rho$ such that $u_{j} \notin$ $H^{1+\epsilon}$ for any $\epsilon>0$.

If $\xi(x)=a \rho(x)$ where $a$ is a positive constant, then we can derive from (1.1)(1.3) another eigenvalue problem which has the same eigenvalues but different eigenfunctions. Let $\sigma=u^{\prime} / \tau$. Then from (1.1) we have

$$
-\sigma^{\prime}+a \rho u=\lambda \rho u \text {. }
$$

Dividing by $\rho$ and differentiating yields

$$
-\left(\sigma^{\prime} / \rho\right)^{\prime}+a \tau \sigma=\lambda \tau \sigma .
$$

We also see that $\sigma$ and $\sigma^{\prime} / \rho$ are in $H^{1}$ and hence satisfy the periodic boundary conditions. Thus, (1.1) and (1.5) have the same eigenvalues and their eigenfunctions are related by $\sigma_{j}=u_{j}^{\prime} / \tau$. Note that the roles of $\tau$ and $\rho$ are interchanged in passing between (1.1) and (1.5). (1.5) has the variational formulation

$$
\int_{0}^{2 \pi} \frac{1}{\rho} \sigma^{\prime} \bar{\zeta}^{\prime} d x+a \int_{0}^{2 \pi} \tau \sigma \bar{\zeta} d x=\lambda \int_{0}^{2 \pi} \tau \sigma \bar{\zeta} d x, \text { for all } \zeta \in H^{1} .
$$

The eigenfunctions $\sigma_{j} \in H^{1}$ but there exists $\rho$ and $\tau$ such that $\sigma_{j} \notin H^{1+\epsilon}$ for any $\epsilon>0$.

Now we consider a variational formulation of (1.1)-(1.3) which is different than (1.4). We introduce the new independent variable $\sigma=u^{\prime} / \tau$ and write (1.1)-(1.3) as a 
system

$$
\begin{aligned}
u^{\prime} & =\tau \sigma, \\
-\sigma^{\prime}+\xi u & =\lambda \rho u, \\
u(0) & =u(2 \pi), \\
\sigma(0) & =\sigma(2 \pi) .
\end{aligned}
$$

Taking the $H^{0}$-inner product of (1.7) with $s$ and (1.8) with $v$ and adding the results, we obtain

$$
\int_{0}^{2 \pi}\left(u^{\prime} \bar{s}-\tau \sigma \bar{s}-\sigma^{\prime} \bar{v}+\xi u \bar{v}\right) d x=\lambda \int_{0}^{2 \pi} \rho u \bar{v} d x .
$$

Here we seek a nonzero pair $u, \sigma$ such that $(1.11)$ holds for all $v, s$. It is easily seen that $(1.11)$ is equivalent to (1.7)-(1.10).

We discuss next a general framework in which to study the variational formulations of eigenvalue problems. Let $H_{1}$ and $H_{2}$ be two Hilbert spaces and let $A(\phi, \psi)$ and $B(\phi, \psi)$ be two bounded sesquilinear forms on $H_{1} \times H_{2}$. We suppose that $A$ satisfies

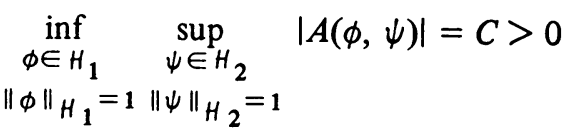

and

$$
\sup _{\phi \in H_{1}}|A(\phi, \psi)|>0, \quad \text { for each nonzero } \psi \in H_{2} \text {. }
$$

A form on $H_{1} \times H_{2}$ which is bounded and satisfies (1.12) and (1.13) will be called a proper form. In addition, we assume a compactness relation between $A$ and $B: T: H_{1}$ $\longrightarrow \mathrm{H}_{2}$ is compact, where $T$ is the operator defined by (cf. Babuška and Aziz [2, p.

$$
A(T \phi, \psi)=B(\phi, \psi), \quad \text { for all } \phi \in H_{1}, \psi \in H_{2} \text {. }
$$

A complex number $\lambda$ is called an eigenvalue of the form $A$ relative to the form $B$ if there is a nonzero eigenvector $\phi \in H_{1}$ such that

$$
A(\phi, \psi)=\lambda B(\phi, \psi), \text { for all } \psi \in H_{2} \text {. }
$$

It is easily seen that $\lambda$ and $\phi$ satisfy (1.15) if and only if $\lambda T \phi=\phi$. If $\lambda$ is an eigenvalue of (1.15), then $\lambda$ is also an eigenvalue of the eigenvalue problem which is adjoint to (1.15), i.e., there exists a nonzero adjoint eigenvector $\psi \in \mathrm{H}_{2}$ such that

$$
A(\phi, \psi)=\lambda B(\phi, \psi), \text { for all } \phi \in H_{1} \text {. }
$$

If we let $H_{1}=H_{2}=H^{1}$ and let

$$
A(u, \zeta)=\int_{0}^{2 \pi} \frac{1}{\tau} u^{\prime} \bar{\zeta}^{\prime} d x+\int_{0}^{2 \pi} \xi u \bar{\zeta} d x
$$

$$
B(u, \zeta)=\int_{0}^{2 \pi} \rho u \bar{\zeta}
$$

we get the variational formulation (1.4). If we let 


$$
\begin{aligned}
& A(\sigma, \zeta)=\int_{0}^{2 \pi} \frac{1}{\rho} \sigma^{\prime} \bar{\zeta}^{\prime} d x+a \int_{0}^{2 \pi} \tau \sigma \bar{\zeta} d x \\
& B(\sigma, \zeta)=\int_{0}^{2 \pi} \tau \sigma \bar{\zeta} d x
\end{aligned}
$$

we get (1.6).

If we let

$$
\begin{aligned}
& A(\phi, \psi)=A(u, \sigma, v, s)=\int_{0}^{2 \pi}\left(u^{\prime} \bar{s}-\tau \sigma \bar{s}-\sigma^{\prime} \bar{v}+\xi u \bar{v}\right) d x \\
& B(\phi, \psi)=B(u, \sigma, v, s)=\int_{0}^{2 \pi} \rho u \bar{v} d x
\end{aligned}
$$

where $\phi$ is the pair of functions $u, \sigma$ and $\psi$ is the pair $v, s$, we get the variational formulation (1.11). In the next section we discuss a class of choices for the spaces $H_{1}$ and $\mathrm{H}_{2}$.

3.2. A Variational Formulation of the Eigenvalue Problem. There are many ways to choose the spaces $H_{1}$ and $H_{2}$ so that the sesquilinear forms $A$ and $B$ defined in (1.20) and (1.21) are bounded and that $A$ is proper. In this subsection we discuss the following class of choices:

$$
\begin{aligned}
& H_{1}=H^{\alpha(x), 0, L} \times H^{\beta(x), 0, L}, \\
& H_{2}=H^{1-\beta(x), 0, L} \times H^{1-\alpha(x), 0, L},
\end{aligned}
$$

where $0<\alpha(x), \beta(x)<1$, i.e., we let $\phi=(u, \sigma) \in H^{\alpha(x), 0, L} \times H^{\beta(x), 0, L}$ and $\psi=$ $(v, s) \in H^{1-\beta(x), 0, L} \times H^{1-\alpha(x), 0, L}$. We now proceed to show that $A$ and $B$ (defined in (1.20) and (1.21)) satisfy the aforementioned conditions.

THEOREM 1. The sesquilinear forms $A$ and $B$ defined in (1.20) and (1.21), respectively, are bounded on $H_{1} \times H_{2}$, with $H_{1}$ and $H_{2}$ defined in (2.1) and (2.2).

Proof. First we consider $B$. Using Theorem 3 in Chapter 2 we have

$$
\begin{aligned}
|B(u, \sigma, v, s)| & =\left|\int_{0}^{2 \pi} \rho u \bar{v} d x\right| \\
& \leqslant C\|u\|_{0}\|v\|_{0} \leqslant C\|u\|_{\alpha, 0, L}\|v\|_{1-\beta, 0, L} \\
& \leqslant C\left(\|u\|_{\alpha, 0, L}^{2}+\|\sigma\|_{\alpha, 0, L}^{2}\right)^{1 / 2}\left(\|v\|_{1-\beta, 0, L}^{2}+\|s\|_{1-\alpha, 0, L}^{2}\right)^{1 / 2} \\
& =C\|(u, \sigma)\|_{H_{1}}\|(v, s)\|_{H_{2}}
\end{aligned}
$$

for all $(u, \sigma) \in H_{1}$ and $(v, s) \in H_{2}$, i.e., $B$ is bounded on $H_{1} \times H_{2}$.

To show that $A$ is bounded we must prove similar inequalities for each of the four terms in $A$. The terms $\int_{0}^{2 \pi} \tau \sigma \bar{s} d x$ and $\int_{0}^{2 \pi} \xi u \bar{v} d x$ are treated in a similar way as $\int_{0}^{2 \pi} \rho u \bar{v} d x$. Now consider $\int_{0}^{2 \pi} u^{\prime} \bar{s} d x$.

Let $u, s \in E$. From Theorem 4 in Chapter 2 we have

$$
\Lambda^{\alpha(x)-1} u^{\prime}=\left(\Lambda^{\alpha(x)-1} u\right)^{\prime}+w
$$

with $\|w\|_{0} \leqslant C(\epsilon)\|u\|_{\alpha_{+}-1+\epsilon}$. Thus 


$$
\begin{aligned}
\left\|\Lambda^{\alpha(x)-1} u^{\prime}\right\|_{0} & \leqslant\left\|\left(\Lambda^{\alpha(x)-1} u\right)^{\prime}\right\|_{0}+C(\epsilon)\|u\|_{\alpha_{+}-1+\epsilon} \\
& \leqslant\left\|\Lambda \Lambda^{\alpha(x)-1} u\right\|_{0}+C(\epsilon)\|u\|_{\alpha_{+}-1+\epsilon}
\end{aligned}
$$

By Theorem 2 in Chapter 2 we have

$$
\Lambda \Lambda^{\alpha(x)-1} u=\Lambda^{\alpha(x)} u+z
$$

with $\|z\|_{0} \leqslant C(\epsilon)\|u\|_{\alpha_{+}-1+\epsilon}$. Thus

$$
\left\|\Lambda \Lambda^{\alpha(x)-1} u\right\|_{0} \leqslant\left\|\Lambda^{\alpha(x)} u\right\|_{0}+C(\epsilon)\|u\|_{\alpha_{+}-1+\epsilon} .
$$

Since $\alpha_{+}<1$ we have $\alpha_{+}-1+\epsilon<0$ for $\epsilon$ sufficiently small and hence by Theorem 3 in Chapter 2,

$$
\|u\|_{\alpha_{+}-1+\epsilon} \leqslant C\left(\left\|\Lambda^{\alpha(x)} u\right\|_{0}+\|u\|_{-L}\right) .
$$

Again by Theorem 3 in Chapter 2 we have

$$
\left\|u^{\prime}\right\|_{-L} \leqslant\|u\|_{-L+1} \leqslant C\left(\left\|\Lambda^{\alpha(x)} u\right\|_{0}+\|u\|_{-L}\right) .
$$

Using Theorem 8 in Chapter 2, we obtain

$$
\left|\int_{0}^{2 \pi} u^{\prime} \bar{s} d x\right| \leqslant C\left\|u^{\prime}\right\|_{\alpha-1,0, L}\|s\|_{1-\alpha, 0, L}
$$

Now, combining this with (2.3)-(2.6), we obtain

$$
\left|\int_{0}^{2 \pi} u^{\prime} \bar{s} d x\right| \leqslant C\|u\|_{\alpha, 0, L}\|s\|_{1-\alpha, 0, L}
$$

From the fact that (2.7) holds for all $u, s \in E$ we see immediately by passage to the limit that it also holds for $u \in H^{\alpha, 0, L}$ and $s \in H^{1-\alpha, 0, L}$; in fact, for $u \in H^{\alpha, 0, L}$ and $s \in H^{1-\alpha, 0, L}$, the expression $\int_{0}^{2 \pi} u^{\prime} s d x$ is defined by such passage to the limit. It follows immediately from (2.7) that

$$
\left|\int_{0}^{2 \pi} u^{\prime} s d x\right| \leqslant C\|(u, \sigma)\|_{H_{1}}\|(v, s)\|_{H_{2}}
$$

for all $(u, \sigma) \in H_{1}$ and $(v, s) \in H_{2}$.

In an analogous way we show that

$$
\left|\int_{0}^{2 \pi} \sigma^{\prime} \bar{v} d x\right| \leqslant C\|(u, \sigma)\|_{H_{1}}\|(v, s)\|_{H_{2}} .
$$

This completes the proof.

THEOREM 2. The sesquilinear form $A$ defined in (1.20) is proper, i.e., it satisfies inequalities (1.12) and (1.13), provided $\beta=1-\alpha$. that

Proof. Given $(u, \sigma) \in H^{\alpha, 0, L} \times H^{\beta, 0, L}$ we seek $(v, s) \in H^{1-\beta, 0, L} \times H^{1-\alpha, 0, L}$ so

$$
A(u, \sigma, v, s) \geqslant C\left(\|u\|_{\alpha, 0, L}^{2}+\|\sigma\|_{\beta, 0, L}^{2}\right)
$$

and

$$
\|v\|_{\alpha, 0, L}+\|s\|_{\beta, 0, L} \leqslant C^{\prime}\left(\|u\|_{\alpha, 0, L}+\|\alpha\|_{\beta, 0, L}\right)
$$

with $C$ and $C^{\prime}$ positive constants. These two inequalities yield inequality (1.12). Since our form $A$ is Hermitian, we also obtain (1.13). This will establish the desired result. We divide the proof into several parts.

(1) Let $u \in H^{\alpha, 0, L}$. We will construct $S \in H^{1-\alpha, 0, L}$ so that 


$$
\begin{aligned}
& \|S\|_{1-\alpha, 0, L} \leqslant C\|u\|_{\alpha, 0, L}, \\
& \int_{0}^{2 \pi} u^{\prime} \bar{S} d x \geqslant C_{1}\|u\|_{\alpha, 0, L}^{2}-C_{2}\|u\|_{0}^{2},
\end{aligned}
$$

with $C, C_{1}>0$ and $C_{2}$ independent of $u$.

It follows from Theorem 8 in Chapter 2 that the sesquilinear form $-\int_{0}^{2 \pi} \phi \bar{y} d x$ is proper on $H^{\alpha, 0, L} \times H^{-\alpha, 0, L}$. It is also easily seen that $\int_{0}^{2 \pi} \Lambda^{\alpha} \phi \overline{\Lambda^{\alpha} u} d x$ defines a bounded linear functional on $H^{\alpha, 0, L}$. Thus there exists (see Babuška and Aziz [2, p. 112]) $y \in H^{-\alpha, 0, L}$ such that

$$
-\int_{0}^{2 \pi} \phi \bar{y}=\int_{0}^{2 \pi} \Lambda^{\alpha} \phi \overline{\Lambda^{\alpha} u} d x
$$

for any $\phi \in H^{\alpha, 0, L}$, and $\|y\|_{-\alpha, 0, L} \leqslant C\|u\|_{\alpha, 0, L}$.

Let $\tilde{y}=y-a_{0}$, where $a_{0}=(1 / 2 \pi) \int_{0}^{2 \pi} y d x$. Clearly

$$
\left|a_{0}\right| \leqslant C\|y\|_{-\alpha, 0, L} \leqslant C\|u\|_{\alpha, 0, L}
$$

and thus

$$
\|\tilde{y}\|_{-\alpha, 0, L} \leqslant C\|u\|_{\alpha, 0, L}
$$

Since the 0 th Fourier coefficient of $\tilde{y}$ is 0 we can find $S \in H^{1-\alpha, 0, L}$ such that $S^{\prime}=\tilde{y}$ and

$$
\|S\|_{1-\alpha, 0, L} \leqslant C\|\tilde{y}\|_{-\alpha, 0, L} \leqslant C\|u\|_{\alpha, 0, L}
$$

$S$ is the primitive of $\tilde{y}$ with 0 th Fourier coefficient equal to 0 .

We return now to $(2.10)$ and set $\phi=u$; this gives

$$
\begin{aligned}
2 \pi\left\|\Lambda^{\alpha(x)} u\right\|_{0}^{2} & =-\int_{0}^{2 \pi} u \bar{y} d x \\
& =-\int_{0}^{2 \pi} u \overline{\widetilde{y}} d x-\bar{a}_{0} \int_{0}^{2 \pi} u d x \\
& =-\int_{0}^{2 \pi} u \bar{S}^{\prime} d x-\bar{a}_{0} \int_{0}^{2 \pi} u d x \\
& =\int_{0}^{2 \pi} u^{\prime} \bar{S} d x-\bar{a}_{0} \int_{0}^{2 \pi} u d x .
\end{aligned}
$$

Using (2.11), we have

$$
\left|\bar{a}_{0} \int_{0}^{2 \pi} u d x\right| \leqslant \theta\|u\|_{\alpha, 0, L}^{2}+C(\theta)\|u\|_{0}^{2}
$$

for arbitrary $\theta$. From (2.13), (2.14) with small $\theta$, and the fact that $\|u\|_{-L} \leqslant\|u\|_{0}$, we get

$$
\int_{0}^{2 \pi} u^{\prime} \bar{S} d x \geqslant C_{1}\|u\|_{\alpha, 0, L}^{2}-C_{2}\|u\|_{0}^{2}
$$

This proves (2.9). Also, (2.12) yields (2.8).

(2) We consider now the complete form $A$ defined in (1.20). Given $u \in H^{\alpha, 0, L}$, $\sigma \in H^{\beta, 0, L}$ we set

$$
v=D u+w, \quad s=S+z,
$$


where $S$ satisfies (2.8) and (2.9) and $z, w$ and the constant $D$ will be determined later. Using (2.15), we have

$$
\begin{aligned}
A(u, \sigma, v, s)= & \int_{0}^{2 \pi} u^{\prime} \bar{S} d x+\int_{0}^{2 \pi} u^{\prime} \bar{z} d z-\int_{0}^{2 \pi} \tau \sigma \bar{S} d x-\int_{0}^{2 \pi} \tau \sigma \bar{z} d x \\
& -D \int_{0}^{2 \pi} \sigma^{\prime} \bar{u} d x-\int_{0}^{2 \pi} \sigma^{\prime} \bar{w} d x \\
& +D \int_{0}^{2 \pi} \xi|u|^{2} d x+\int_{0}^{2 \pi} \xi u \bar{w} d x .
\end{aligned}
$$

For $D$ sufficiently large (depending on $C_{2}$ in (2.9) and on $\xi$ ) we have

$$
D \int_{0}^{2 \pi} \xi|u|^{2} d x \geqslant 2 C_{2}\|u\|_{0}^{2} \text {. }
$$

Choosing $w=z^{\prime} / \xi$, we can write

$$
\int_{0}^{2 \pi} u^{\prime} \bar{z} d x+\int_{0}^{2 \pi} u \xi \bar{w} d x=\int_{0}^{2 \pi} u\left(-\bar{z}^{\prime}+\xi \bar{w}\right) d x=0
$$

Combining (2.9), (2.16)-(2.18), we get

(2.19) $\quad A(u, \sigma, v, s) \geqslant C\|u\|_{\alpha, 0, L}^{2}+\int_{0}^{2 \pi} \sigma\left(-\tau \bar{S}-\tau \bar{z}+D \bar{u}^{\prime}+\left(\bar{z}^{\prime} / \xi\right)^{\prime}\right) d x$.

(3) Using Theorem 8 in Chapter 2, we see that we can find $V \in H^{-\beta, 0, L}$ so that

$$
\int_{0}^{2 \pi} \sigma \bar{V} d x \geqslant C\|\sigma\|_{\beta, 0, L}^{2}
$$

and

$$
\|V\|_{-\beta, 0, L}=\|\sigma\|_{\beta, 0, L}
$$

Next, we show that we can find $z$ so that

$$
\int_{0}^{2 \pi} \phi\left(-\tau \bar{S}-\tau \bar{z}+D \bar{u}^{\prime}+\left(\bar{z}^{\prime} / \xi\right)^{\prime}\right) d x=\int_{0}^{2 \pi} \phi \bar{V} d x
$$

for all $\phi \in H^{\beta(x), 0, L}$, and thus in particular for $\phi=\sigma$. Consider the following linear functional on $\phi \in H^{1}$ :

$$
\int_{0}^{2 \pi} \phi \bar{V} d x+\int_{0}^{2 \pi} \tau \phi \bar{S}-D \int_{0}^{2 \pi} \phi \bar{u}^{\prime} d x
$$

Clearly

$$
\begin{aligned}
& \left|\int_{0}^{2 \pi} \phi \bar{V} d x\right| \leqslant C\|\phi\|_{1}\|V\|_{-1} \leqslant C\|\phi\|_{1}\|V\|_{-\beta, 0, L}, \\
& \left|\int_{0}^{2 \pi} \tau \phi \bar{S} d x\right| \leqslant C\|\phi\|_{1}\|S\|_{0} \\
& \left|\int_{0}^{2 \pi} \phi \bar{u}^{\prime} d x\right| \leqslant C\|\phi\|_{1}\|u\|_{0} \leqslant C\|\phi\|_{1}\|u\|_{\alpha, 0, L} .
\end{aligned}
$$

Thus, (2.23) defines a bounded linear functional on $H^{1}$. Now consider the following sesquilinear form on $H^{1} \times H^{1}$ :

$$
-\int_{0}^{2 \pi} \tau \phi \bar{z} d x+\int_{0}^{2 \pi} \phi\left(\bar{z}^{\prime} / \xi\right)^{\prime} d x .
$$

Since 


$$
\begin{gathered}
\left|\int_{0}^{2 \pi} \tau \phi \bar{z} d x\right| \leqslant C\|\phi\|_{0}\|z\|_{0} \leqslant C\|\phi\|_{1}\|z\|_{1}, \\
\left|\int_{0}^{2 \pi} \phi(\vec{z} / \xi)^{\prime} d x\right|=\left|\int_{0}^{2 \pi} \frac{1}{\xi} \phi^{\prime} \vec{z} d x\right| \leqslant C\|\phi\|_{1}\|z\|_{1}
\end{gathered}
$$

and

$$
\begin{aligned}
& \left.\left|-\int_{0}^{2 \pi} \tau\right| \phi\right|^{2} d x+\int_{0}^{2 \pi} \phi\left(\bar{\phi}^{\prime} / \xi\right)^{\prime} d x \mid \\
& \quad=\int_{0}^{2 \pi} \tau|\phi|^{2} d x+\int_{0}^{2 \pi} \frac{1}{\xi}\left|\phi^{\prime}\right|^{2} d x \geqslant C\|\phi\|_{1}^{2},
\end{aligned}
$$

we see that (2.27) is a proper form on $H^{1} \times H^{1}$. Hence (see Babuska and Aziz [2, p. 112]) there exists $z \in H^{1}$ such that (2.22) holds for all $\phi \in H^{1}$ and (cf. (2.24), (2.25) and (2.26))

$$
\|z\|_{1} \leqslant C\left(\|V\|_{-\beta, 0, L}+\|S\|_{0}+\|u\|_{\alpha, 0, L}\right) .
$$

Using (2.8) and (2.21), we thus have

$$
\|z\|_{1} \leqslant C\left(\|u\|_{\alpha, 0, L}+\|\sigma\|_{\beta, 0, L}\right)
$$

Since (2.22) is valid for all $\phi \in H^{1}$, we see that

$$
w^{\prime}=\left(z^{\prime} / \xi\right)^{\prime}=V+\tau S+\tau z-D u^{\prime} .
$$

Write $w=a_{0}(w)+\left(w-a_{0}(w)\right)$, where $a_{0}$ is the 0th Fourier coefficient of $w$. From (2.28) and the definition of $w$ we have

$$
\left|a_{0}(w)\right| \leqslant\left|\frac{1}{2 \pi} \int_{0}^{2 \pi} \frac{1}{\xi} z^{\prime} d x\right| \leqslant C\|z\|_{1} \leqslant C\left(\|u\|_{\alpha, 0, L}+\|\sigma\|_{\beta, 0, L}\right) .
$$

Also, using Theorem 3 of Chapter 2, we see that

$$
\begin{aligned}
\left\|w-a_{0}(w)\right\|_{\alpha, 0, L} & \leqslant\left\|V+\tau S+\tau z-D u^{\prime}\right\|_{\alpha-1,0, L} \\
& \leqslant C\left(\|V\|_{\alpha-1,0, L}+\|S\|_{0}+\|z\|_{0}+\left\|\Lambda^{\alpha-1} u^{\prime}\right\|_{0}+\|u\|_{-L+1}\right)
\end{aligned}
$$

Using Theorems 2, 3 and 4 of Chapter 2, we get

$$
\left\|\Lambda^{\alpha-1} u^{\prime}\right\|_{0}+\|u\|_{-L+1} \leqslant C\|u\|_{\alpha, 0, L} .
$$

From (2.8), (2.21), (2.28), (2.30)-(2.32), and the fact that $\beta=1-\alpha$ we have

$$
\|w\|_{\alpha, 0, L} \leqslant C\left(\|u\|_{\alpha, 0, L}+\|\sigma\|_{\beta, 0, L}\right) .
$$

We have shown that (2.22) holds for all $\phi \in H^{1}$. (2.33) shows that $w^{\prime}=\left(z^{\prime} / \xi\right)^{\prime} \in$ $H^{-\beta(x), 0, L}$, and thus $-\tau S-\tau z+D u^{\prime}+\left(z^{\prime} / \xi\right)^{\prime}$ and $V$ are in $H^{-\beta(x), 0, L}$. It thus follows by taking limits that (2.22) holds for all $\phi \in H^{\beta(x), 0, L}$.

(4) We have now chosen each of the functions introduced in (2.15). With these choices for $v$ and $s$ we see from (2.19), (2.22) and (2.20) that

$$
A(u, \sigma, v, s) \geqslant C\|u\|_{\alpha, 0, L}^{2}+\int_{0}^{2 \pi} \sigma \bar{V} d x \geqslant C\left(\|u\|_{\alpha, 0, L}^{2}+\|\sigma\|_{\beta, 0, L}^{2}\right)
$$


From (2.15) and (2.33) we have

$$
\|v\|_{1-\beta, 0, L} \leqslant C\left(\|u\|_{\alpha, 0, L}+\|\sigma\|_{\beta, 0, L}\right)
$$

and from (2.15), (2.8) and (2.28) we have

$$
\|s\|_{1-\alpha, 0, L} \leqslant C\left(\|u\|_{\alpha, 0, L}+\|\sigma\|_{\beta, 0, L}\right) \text {. }
$$

The inequalities (2.34)-(2.36) yield the proof of (1.12).

Finally, we note that under our present hypotheses we have $H_{1}=H_{2}$ and $\overline{A(v, s, u, \sigma)}=A(u, \sigma, v, s)$, i.e., $A$ is Hermitian. Thus, (1.13) follows from (1.12). This completes the proof of Theorem 2 .

We end this section by observing that the operator $T: H_{1} \rightarrow H_{1}$ defined by $A(T \phi, \psi)=B(\phi, \psi)$ for $\phi \in H_{1}, \psi \in H_{2}$ is compact (cf.(1.14)). To see this, we first note that $T$ is a bounded operator from $H_{1}$ to $H^{1} \times H^{1}$, and then apply Theorem 7 in Chapter 2. (Cf. Theorem 10 in Section 3.4.)

3.3. The Approximate Eigenvalue Problem. In this section we associate an approximate eigenvalue problem with (1.15). Let $M_{1}^{h} \subset H_{1}$ and $M_{2}^{h} \subset H_{2}$, where either $0<h \leqslant 1$ or $h=h_{N}, h_{N} \downarrow 0$, be given families of finite dimensional subspaces. We assume conditions analogous to (1.12) and (1.13):

$$
\inf _{\substack{\phi \in M_{1}^{h} \\\|\phi\|_{H_{1}}=1}} \sup _{\substack{\psi \in M_{2}^{h} \\\|\psi\|_{H_{2}}=1}}|A(\phi, \psi)| \geqslant C h^{\delta}, \quad C>0 \text { and } \delta \geqslant 0 \text { independent of } h,
$$

and

$$
\sup _{\phi \in M_{1}^{h}}|A(\phi, \psi)|>0, \quad \text { for each nonzero } \psi \in M_{2}^{h} ;
$$

(3.1) and (3.2) are assumed to hold for $h$ sufficiently small. In addition, we make the convergence assumption

$$
\left\|T_{h}-T\right\| \underset{h \rightarrow 0}{\rightarrow} 0
$$

where $T_{h}: H_{1} \rightarrow H_{1}$ is the operator defined by

$$
\left\{\begin{array}{l}
T_{h} \phi \in M_{1}^{h}, \\
A\left(T_{h} \phi, \psi\right)=B(\phi, \psi), \text { for all } \phi \in H_{1}, \psi \in M_{2}^{h},
\end{array}\right.
$$

$T$ is defined by (1.14), and $\|\cdot\|$ denotes the operator norm on $H_{1}$. We now seek numbers $\lambda^{h}$ for which there exists nonzero $\phi^{h} \in M_{1}^{h}$ such that

$$
A\left(\phi^{h}, \psi\right)=\lambda^{h} B\left(\phi^{h}, \psi\right), \quad \text { for all } \psi \in M_{2}^{h} .
$$

The eigenvalues $\lambda^{h}$ of (3.4) are then used as approximations to the eigenvalues $\lambda$ of (1.15). The eigenvalue problem (3.4) is obviously equivalent to a matrix eigenvalue problem.

The approximations $\lambda^{h}$ defined by (3.4) will be called the Ritz-Galerkin approximations based on the sesquilinear forms $A$ and $B$ and this approximation method will be referred to as the Ritz-Galerkin method based on the forms $A$ and $B$. 
The formulations (1.15) and (3.4) cover a wide variety of eigenvalue problems and approximation methods. A full discussion of the associated convergence results involves several notions from the theory of nonselfadjoint operators such as ascent, algebraic multiplicity, and generalized eigenvectors. In all of our applications $H_{1}=H_{2}, A$ is Hermitian, and $M_{1}^{h}=M_{2}^{h}$. Furthermore, using the fact that $A(u, \sigma, u, \sigma)>0$ for $(u, \sigma)$ an eigenvector, we can easily show that our problem has no generalized eigenvectors. We shall thus be able to use a restricted form of the general convergence theorem.

For the remainder of the paper we suppose $\alpha+\beta=1,0<\alpha(x)<1$, and $M_{1}^{h}=$ $M_{2}^{h}=M^{h}$. Then $H_{1}=H_{2}=H$ and $A$ is a Hermitian form on $H$. Let $\lambda_{0}$ be an eigenvalue of (1.15) with algebraic and geometric multiplicity $m$. Then $m$ eigenvalues of (3.4) converge to $\lambda_{0}$ as $h \rightarrow 0$; let $\lambda_{0}^{h}$ be any one of these eigenvalues. Let $N_{0}$ be the $m$ dimensional space of eigenvectors of (1.15) corresponding to $\lambda_{0}$. We introduce the notation

$$
\epsilon^{h}=\sup _{\substack{\phi_{0} \in N_{0} \\\left\|\phi_{0}\right\|_{H}=1}} \inf _{x \in M^{h}}\|\phi-\chi\|_{H} .
$$

The rate of convergence of $\lambda_{0}^{h}$ to $\lambda_{0}$ (as well as the rate of convergence of the corresponding eigenvector approximation) is estimated in the following

THEOREM 3. There is a constant $C$, independent of $h$, such that

$$
\left|\lambda_{0}-\lambda_{0}^{h}\right| \leqslant C h^{-\delta}\left(\epsilon^{h}\right)^{2}
$$

with $\delta$ as in (3.1). If $\phi_{0}^{h}$ is an eigenvector of (3.4) corresponding to $\lambda_{0}^{h}$ with $\left\|\phi_{0}^{h}\right\|_{H}=1$, then for each $h$ there is an eigenvector $\widetilde{\phi}_{0}^{h}$ of $(1.15)$ corresponding to $\lambda_{0}$ such that

$$
\left\|\widetilde{\phi}_{0}^{h}-\phi_{0}^{h}\right\|_{H} \leqslant C h^{-\delta} \epsilon^{h} \text {. }
$$

For a proof of the general rate of convergence theorem for the case in which the ascent is one (which includes the above theorem) we refer to Babuška and Aziz [2] and Fix [9]. For a proof in the case where the ascent is greater than one we refer to Kolata [11] These references treat the case $\delta=0$. An obvious modification of the proof yields the case $\delta>0$. See also Bramble and Osborn [5] and Osborn [22].

We now suppose that we have two families of spaces $\left\{S_{1}^{h}\right\}$ and $\left\{S_{2}^{h}\right\}$ satisfying the following properties:

$$
\begin{aligned}
& S_{j}^{h} \subset H^{k_{j}}, \quad j=1,2, \text { with } k_{j} \text { constant, } k_{j} \geqslant 1 ; \\
& 1 \in S_{1}^{h}, \quad j=1,2, \text { and }\left(S_{1}^{h}\right)^{\prime}=\left\{v \in S_{2}^{h}: \int_{0}^{2 \pi} v d x=0\right\},
\end{aligned}
$$

where $\left(S_{1}^{h}\right)^{\prime}$ denotes the set of derivatives of all functions in $S_{1}^{h}$;

$$
\inf _{\chi \in S_{j}^{h}}\|u-\chi\|_{\gamma(x), 0, L} \leqslant C(\epsilon) h^{\mu-\epsilon}\|u\|_{\gamma(x), \mu, L}, \quad j=1,2,
$$

where $0 \leqslant \gamma(x)<k_{j}, \gamma_{+}-\gamma_{-}<1,0 \leqslant \mu, \gamma_{+}+\mu \leqslant t_{j}$ with $t_{j} \geqslant 1, \epsilon>0$; if $\gamma(x)=\gamma$ is constant then we can let $\epsilon=0$;

$$
\left\|p_{h} u\right\|_{\gamma(x), 0, L} \leqslant C h^{-\delta}\|u\|_{\gamma(x), 0, L},
$$

where $p_{h}$ is the $H^{0}$-orthogonal projection of $u$ onto $S_{2}^{h}, 0 \leqslant \gamma(x) \leqslant k_{2}, \gamma_{+}-\gamma_{-}<1$ 
and $\delta=\delta(\gamma)$ is a nonnegative constant depending on $\gamma$; for the case $\gamma(x)=$ constant, one may choose $\delta(\gamma)=0$.

Then we define

$$
M^{h}=S_{1}^{h} \times S_{2}^{h}
$$

We will discuss assumption (3.3) in Section 3.4. We now show that (3.1) and (3.2) are satisfied.

THEOREM 4. Suppose $S_{1}^{h}$ and $S_{2}^{h}$ satisfy assumptions (3.8)-(3.11). Then, with $M^{h}$ defined in (3.12), assumptions (3.1) and (3.2) hold with $\delta=\delta(\beta)$ as in assumption (3.11).

Proof. The proof of this theorem parallels that of Theorem 2. Given $(u, \sigma) \in$ $S_{1}^{h} \times S_{2}^{h}$ we seek $(v, s) \in S_{1}^{h} \times S_{2}^{h}$ so that

and

$$
A(u, \sigma, v, s) \geqslant C\left(\|u\|_{\alpha, 0, L}^{2}+\|\sigma\|_{\beta, 0, L}^{2}\right)
$$

$$
\|v\|_{\alpha, 0, L}+\|s\|_{\beta, 0, L} \leqslant C^{\prime} h^{-\delta}\left(\|u\|_{\alpha, 0, L}+\|\alpha\|_{\beta, 0, L}\right)
$$

with $C$ and $C^{\prime}$ positive constants that do not depend on $h$, with $\delta=\delta(\beta)$ as in assumption (3.11). These inequalities yield (3.1). (3.2) follows from the fact that $A$ is Hermitian. We divide the proof into several parts.

(1) Let $u \in S_{1}^{h}, \sigma \in S_{2}^{h}$. In the proof of Theorem 2 we constructed $S$ satisfying (2.8) and (2.9). Let $\hat{S}$ be the $H^{0}$-orthogonal projection of $S$ onto $S_{2}^{h}$. From (3.11) and (2.8) we have

$$
\|\hat{S}\|_{1-\alpha, 0, L} \leqslant C h^{-\delta}\|S\|_{1-\alpha, 0, L} \leqslant C h^{-\delta}\|u\|_{\alpha, 0, L},
$$

where $\delta=\delta(\beta)$. Since from (3.9) we have $u^{\prime} \in S_{2}^{h}$, we see that

$$
\int_{0}^{2 \pi} u^{\prime}(\bar{S}-\overline{\hat{S}}) d x=0 .
$$

Thus, from (2.9) we have

$$
\int_{0}^{2 \pi} u^{\prime} \overline{\hat{S}} d x \geqslant C_{1}\|u\|_{\alpha, 0, L}^{2}-C_{2}\|u\|_{0}^{2} .
$$

(2) With $S$ replaced by $\hat{S}$ we next construct $z, w$ and $D$ exactly as in the proof of Theorem 2. We have $w=z^{\prime} / \xi$ and (cf. (2.22))

$$
\int_{0}^{2 \pi} \sigma\left(-\tau \overline{\widehat{S}}-\tau \bar{z}+D u^{\prime}+\left(\bar{z}^{\prime} / \xi\right)^{\prime}\right) d x=\int_{0}^{2 \pi} \sigma \bar{V} d x,
$$

where $V$ satisfies $(2.20)$ and (2.21). We also note that

$$
\begin{aligned}
\|z\|_{1} & \leqslant C\left(\|u\|_{\alpha, 0, L}+\|\sigma\|_{\beta, 0, L}\right), \\
\|w\|_{\alpha, 0, L} & \leqslant C\left(\|u\|_{\alpha, 0, L}+\|\sigma\|_{\beta, 0, L}\right) .
\end{aligned}
$$

These results are seen to hold by observing that replacing $S$ by $\hat{S}$ does not alter inequalities (2.28) and (2.33).

(3) Consider now the sesquilinear form $\int_{0}^{2 \pi} \phi \bar{\psi} d x$. If follows from Theorem 8 in Chapter 2 that this form is proper on $H^{1-\alpha, 0, L} \times H^{\alpha-1,0, L}$. Let $\psi \in S_{2}^{h}$. Then, using Theorem 9 in Chapter 2, we can find $\phi \in H^{1-\alpha, 0, L}$ such that 


$$
\int_{0}^{2 \pi} \phi \bar{\psi} d x \geqslant C\|\psi\|_{\alpha-1,0, L}^{2}
$$

with $C>0$ and independent of $h$, and

$$
\|\phi\|_{1-\alpha, 0, L}=\|\psi\|_{\alpha-1,0, L} .
$$

Let $\hat{\phi}=p_{h} \phi$, where $p_{h} \phi$ is the $H^{0}$-orthogonal projection of $\phi$ onto $S_{2}^{h}$. Then, from (3.18) we have

$$
\int_{0}^{2 \pi} \hat{\phi} \bar{\psi} d x \geqslant C\|\psi\|_{\alpha-1,0, L}^{2}
$$

and using (3.19) and (3.11), we obtain

$$
\|\hat{\phi}\|_{1-\alpha, 0, L} \leqslant C h^{-\delta}\|\phi\|_{1-\alpha, 0, L}=C h^{-\delta}\|\psi\|_{\alpha-1,0, L}
$$

where $\delta=\delta(\beta)$. Combining (3.20) and (3.21) we find

$$
\inf _{\substack{\psi \in S_{2}^{h} \\\|\psi\|_{\alpha-1,0, L}=1} \sup _{\phi \in S_{2}^{h}} \mid\left\|_{0}\right\|_{1-\alpha, 0, L}=1}|\bar{\psi} d x| \geqslant C h^{\delta} .
$$

We also see that

$$
\sup _{\psi \in S_{2}^{h}}\left|\int_{0}^{2 \pi} \phi \bar{\psi} d x\right|>0, \text { for any nonzero } \phi \in S_{2}^{h} .
$$

Since $w^{\prime} \in H^{\alpha-1,0, L}$, it is easily seen that $\int_{0}^{2 \pi} \phi \bar{w}^{\prime} d x$ is a bounded linear functional on $H^{1-\alpha, 0, L}$.

It thus follows (see Babuška-Aziz [2, pp. 112, 186, 187]) from (3.22) and (3.23) that there exists a unique $w_{1} \in S_{2}^{h}$ such that

and

$$
\int_{0}^{2 \pi} \phi \bar{w}_{1} d x=\int_{0}^{2 \pi} \phi \bar{w}^{\prime} d x, \text { for all } \phi \in S_{2}^{h}
$$

$$
\left\|w_{1}\right\|_{\alpha-1,0, L} \leqslant C h^{-\delta}\left\|w^{\prime}\right\|_{\alpha-1,0, L} \leqslant C h^{-\delta}\|w\|_{\alpha, 0, L} .
$$

Letting $\phi=1$ in (3.24) shows that $\int_{0}^{2 \pi} w_{1} d x=0$. Using (3.9), we see that we can find $\widetilde{w} \in S_{1}^{h}$ such that $(\widetilde{w})^{\prime}=w_{1}$ and $\int_{0}^{2 \pi} \widetilde{w} d x=0$. Now set $\hat{w}=\widetilde{w}+\int_{0}^{2 \pi} w d x$. Then, $(\hat{w})^{\prime}=w_{1}, \int_{0}^{2 \pi} \hat{w} d x=\int_{0}^{2 \pi} w d x$ and, from (3.25),

$$
\begin{aligned}
\|\hat{w}\|_{\alpha, 0, L} & =\left\|\widetilde{w}+\int_{0}^{2 \pi} w d x\right\|_{\alpha, 0, L} \\
& \leqslant\left\|\hat{w}^{\prime}\right\|_{\alpha-1,0, L}+C\|w\|_{0} \leqslant C h^{-\delta}\|w\|_{\alpha, 0, L} .
\end{aligned}
$$

Let $-1<\nu<\alpha_{-}-1$. We easily see that the form $\int_{0}^{2 \pi} \phi \bar{\psi} d x$ is proper on $H^{\nu} \times$ $H^{-\nu}$. We can further prove that

$$
\inf _{\substack{\psi \in S_{2}^{h} \\\|\psi\|_{\nu}=1 \quad \sup _{\phi \in S_{2}^{h}}\|\phi\|_{-\nu}=1}}\left|\int_{0}^{2 \pi} \phi \bar{\psi} d x\right| \geqslant C
$$

and

$$
\sup _{\psi \in S_{2}^{h}}\left|\int_{0}^{2 \pi} \phi \bar{\psi} d x\right|>0, \quad \text { for any nonzero } \phi \in S_{2}^{h}
$$


(cf. (3.22) and (3.23)). Since $w^{\prime} \in H^{\nu}$, we see (Babuška-Aziz [2, pp. 112, 186, 187]) from (3.27) and (3.28) that there is a unique $w_{2} \in S_{2}^{h}$ such that

$$
\int_{0}^{2 \pi} \phi \bar{w}_{2} d x=\int_{0}^{2 \pi} \phi \bar{w}^{\prime} d x \text {, for all } \phi \in S_{2}^{h}
$$

and

$$
\left\|w^{\prime}-w_{2}\right\|_{\nu} \leqslant C \inf _{\chi \in S_{2}^{h}}\left\|w^{\prime}-\chi\right\|_{\nu}
$$

It is immediate from (3.24) and (3.29) that $\hat{w}^{\prime}=w_{2}$. We can thus use (3.30) to estimate $\|w-\hat{w}\|_{0}$. From (3.30), (3.10) and Theorem 3 in Chapter 2 we have

$$
\begin{aligned}
& \|w-\hat{w}\|_{0} \leqslant\|w-\hat{w}\|_{\nu+1} \leqslant C\left\|w^{\prime}-\hat{w}^{\prime}\right\|_{\nu} \leqslant C \text { inf }\left\|w^{\prime}-\chi\right\|_{\nu} \\
& x \in s_{2}^{h} \\
& \leqslant C \inf _{\chi \in\left(s_{1}^{h}\right)^{\prime}}\left\|w^{\prime}-\chi\right\|_{\nu}=C \inf _{\omega \in s_{1}^{h}}\left\|w^{\prime}-\omega^{\prime}\right\|_{\nu} \\
& =C \inf _{\omega \in S_{1}^{h}}\|w-\omega\|_{\nu+1} \leqslant C h^{\tau}\|w\|_{\alpha(x), 0, L},
\end{aligned}
$$

where $0<\tau$ and $\nu+1+\tau<\alpha_{-}$.

(4) Next set $\hat{z}=p_{h} z$, where $p_{h} z$ is the $H^{0}$-orthogonal projection of $z$ onto $S_{2}^{h}$.

With $\hat{w}, \hat{z}$ and $D$ so chosen we set

$$
v=D u+\hat{w}, \quad s=\hat{S}+\hat{z} .
$$

Then $v \in S_{1}^{h}$ and $s \in S_{2}^{h}$. Using (3.32), we have

$$
\begin{aligned}
A(u, \sigma, v, s)=\int_{0}^{2 \pi} u^{\prime} \overline{\hat{S}} d x+\int_{0}^{2 \pi} u^{\prime} \overline{\hat{z}} d x-\int_{0}^{2 \pi} \tau \sigma \overline{\hat{S}} d x \\
\quad-\int_{0}^{2 \pi} \tau \sigma \overline{\hat{z}} d x-D \int_{0}^{2 \pi} \sigma^{\prime} \bar{u} d x-\int_{0}^{2 \pi} \sigma^{\prime} \overline{\hat{w}} d x \\
+D \int_{0}^{2 \pi} \xi|u|^{2} d x+\int_{0}^{2 \pi} \xi u \overline{\hat{w}} d x .
\end{aligned}
$$

From (3.14) and (2.17) we get

$$
\int_{0}^{2 \pi} u^{\prime} \overline{\hat{S}} d x+D \int_{0}^{2 \pi} \xi|u|^{2} d x \geqslant C_{1}\|u\|_{\alpha, 0, L}^{2}
$$

Using (3.24), (3.15) and (2.20) we have

$$
\begin{aligned}
& -\int_{0}^{2 \pi} \tau \sigma \overline{\hat{S}} d x-\int_{0}^{2 \pi} \tau \sigma \bar{z} d x-D \int_{0}^{2 \pi} \sigma^{\prime} \bar{u} d x-\int_{0}^{2 \pi} \sigma^{\prime} \overline{\hat{w}} d x \\
& =\left\{-\int_{0}^{2 \pi} \tau \sigma \overline{\hat{S}} d x-\int_{0}^{2 \pi} \tau \sigma \bar{z} d x-D \int_{0}^{2 \pi} \sigma^{\prime} \bar{u} d x-\int_{0}^{2 \pi} \sigma^{\prime} \bar{w} d x\right\} \\
& \quad+\int_{0}^{2 \pi} \tau \sigma(\bar{z}-\bar{z}) d x \\
& =\int_{0}^{2 \pi} \sigma \bar{V} d x+\int_{0}^{2 \pi} \tau \sigma(\bar{z}-\overline{\hat{z}}) d x \geqslant C\|\sigma\|_{\beta, 0, L}^{2}+\int_{0}^{2 \pi} \tau \sigma(\bar{z}-\overline{\hat{z}}) d x
\end{aligned}
$$


Since $u^{\prime} \in S_{2}^{h}$ we see that

$$
\int_{0}^{2 \pi} u^{\prime} \overline{\hat{z}} d x=\int_{0}^{2 \pi} u^{\prime} \bar{z} d x
$$

Now, combining (3.33)-(3.36), we have

$$
\begin{aligned}
A(u, \sigma, v, s) \geqslant & C\|u\|_{\alpha, 0, L}^{2}+C\|\sigma\|_{\beta, 0, L}^{2} \\
& -\left|\int_{0}^{2 \pi} \tau \sigma(\bar{z}-\overline{\hat{z}}) d x\right|-\left|\int_{0}^{2 \pi} u \xi\left(\overline{\hat{w}}-\frac{\bar{z}}{\xi}\right) d x\right| .
\end{aligned}
$$

From (3.31) and (3.17),

$$
\begin{aligned}
\left|\int_{0}^{2 \pi} u \xi(\overline{\hat{w}}-\bar{w}) d x\right| & \leqslant C\|u\|_{0}\|\hat{w}-w\|_{0} \\
& \leqslant C\|u\|_{0} h^{\tau}\|w\|_{\alpha, 0, L} \leqslant C h^{\tau}\left(\|u\|_{\alpha, 0, L}^{2}+\|\sigma\|_{\beta, 0, L}^{2}\right) .
\end{aligned}
$$

Similarly, from (3.10) and (3.16) we have

$$
\left|\int_{0}^{2 \pi} \tau \sigma(\bar{z}-\overline{\hat{z}}) d x\right| \leqslant C\|\sigma\|_{0}\|z\|_{1} h^{\eta} \leqslant C\left(\|u\|_{\alpha, 0, L}^{2}+\|\sigma\|_{\beta, 0, L}^{2}\right) h^{\eta},
$$

where $0<\eta$. Combining (3.37)-(3.39), we have

$$
A(u, \sigma, v, s) \geqslant \frac{C}{2}\left(\|u\|_{\alpha, 0, L}^{2}+\|\sigma\|_{\beta, 0, L}^{2}\right)
$$

for $h$ sufficiently small with $C>0$ and independent of $h$.

(5) It remains to estimate $\|v\|_{\alpha, 0, L}$ and $\|s\|_{\beta, 0, L}$. From (3.32), (3.26) and (3.17) we have

$$
\begin{aligned}
\|v\|_{\alpha, 0, L} & \leqslant C\left(\|u\|_{\alpha, 0, L}+\|\hat{w}\|_{\alpha, 0, L}\right) \\
& \leqslant C\left(\|u\|_{\alpha, 0, L}+h^{-\delta}\|w\|_{\alpha, 0, L}\right) \leqslant C h^{-\delta}\left(\|u\|_{\alpha, 0, L}+\|\sigma\|_{\beta, 0, L}\right)
\end{aligned}
$$

and, using (3.32), (3.13), (3.11) and (3.16), we have

$$
\|s\|_{\beta, 0, L} \leqslant\|\hat{S}\|_{\beta, 0, L}+\|\hat{z}\|_{\beta, 0, L} \leqslant C h^{-\delta}\left(\|u\|_{\alpha, 0, L}+\|\sigma\|_{\beta, 0, L}\right),
$$

where $\delta=\delta(\beta)$. Finally, combining (3.40)-(3.42), we find

$$
\inf _{\substack{(u, \sigma) \in M^{h} \\\|(u, \sigma)\|_{H}=1}}^{\sup _{(v, s) \in M^{h}} \mid(v, s) \|_{H}=1}
$$

with $C>0, C$ independent of $h$ and $\delta=\delta(\beta)$ in assumption (3.11). This is assumption (3.1). (3.2) follows from this since $A$ is Hermitian. This completes the proof of Theorem 4.

We now consider two specific choices for the spaces $S_{1}^{h}$ and $S_{2}^{h}$.

(a) Trigonometric polynomials. Let $h=h_{N}=1 / N$ and define

$$
S_{1}^{N}=S_{2}^{N}=S^{N}=\operatorname{span}\left\{e^{i k x}\right\}_{k=-N}^{N}
$$

(Here we write $S_{1}^{N}$ and $\lambda_{0}^{N}$ instead of $S_{1}^{1 / N}$ and $\lambda_{0}^{1 / N}$, etc.) We now show that assumptions (3.8)-(3.11) are satisfied for this choice of spaces. (3.8), with any $k_{j} \geqslant 1$, and (3.9) are immediate. 
The basic approximation property of $S^{N}$ with respect to constant order norms is given by

$$
\inf _{\chi \in S}\|u-\chi\|_{\gamma} \leqslant C N^{-\mu}\|u\|_{\gamma+\mu}
$$

for any real $\gamma$ and $\mu$ with $\mu \geqslant 0$, where $p_{N} u$ is the $H^{0}$-orthogonal projection of $u$ onto $S^{N}$. For variable order norms we have the following

THEOREM 5. Suppose $\gamma \in E_{R}$ and $\mu$ is a constant which satisfy $0 \leqslant \gamma(x), \gamma_{+}-$ $\gamma_{-}<1$ and $0 \leqslant \mu \leqslant 1$. Then for each $\epsilon>0$ there is a constant $C(\epsilon)$ such that

$$
\inf _{\chi \in S^{N}}\|u-\chi\|_{\gamma, 0, L} \leqslant \frac{C(\epsilon)}{N^{\mu-\epsilon}}\|u\|_{\gamma, \mu, L}
$$

for all $u \in H^{\gamma, \mu, L}$ and all $N$.

We omit the proof of this theorem (which can be based on (3.43) and an application of Fejer sums) because it will not play an essential role in the applications treated in Section 3.4. In this regard see Theorem 12.

Theorem 5 shows that assumption (3.10) is satisfied with $t_{1}=t_{2}=1+\gamma_{+}$. We note that if $\gamma$ is constant, then we can take $t_{1}=t_{2}=\infty$.

Next we verify (3.11) with $\delta=\gamma_{+}-\gamma_{-}+\epsilon$, where $\epsilon$ is any positive number.

Theorem 6. Suppose $\gamma \in E_{R}$ with $0 \leqslant \gamma(x)$ and $\gamma_{+}-\gamma_{-}<1$. For any $\epsilon>0$ there is a constant $C(\epsilon)$ such that

$$
\left\|p_{N} u\right\|_{\gamma(x), 0, L} \leqslant C(\epsilon) N^{\gamma_{+}{ }^{-\gamma_{-}+\epsilon}\|u\|_{\gamma(x), 0, L}}
$$

for all $u \in H^{\gamma(x), 0, L}$, where $p_{N} u$ is the $H^{0}$-orthogonal projection of $u$ onto $S^{N}$.

Proof. We first note that

$$
\|p\|_{\kappa} \leqslant C N^{\nu}\|p\|_{\kappa-\nu}
$$

for any $p \in S^{N}$, and

$$
\left\|p_{N} \sigma\right\|_{\kappa} \leqslant\|\sigma\|_{\kappa}
$$

where $k$ and $\nu \geqslant 0$ are constants. Using these inequalities and Theorems 1 and 3 in Chapter 2, we have

$$
\begin{aligned}
\left\|p_{N} u\right\|_{\gamma(x), 0, L} & \leqslant C\left(\left\|p_{N} u\right\|_{\gamma_{+}+\epsilon / 2}+\left\|p_{N} u\right\|_{-L}\right) \\
& \leqslant C\left(N^{\gamma_{+}-\gamma_{-}+\epsilon}\left\|p_{N} u\right\|_{\gamma_{-}-\epsilon / 2}+\left\|p_{N} u\right\|_{-L}\right) \\
& \leqslant C\left(N^{\gamma_{+}-\gamma_{-}+\epsilon}\|u\|_{\gamma_{-}-\epsilon / 2}+\|u\|_{-L}\right) \leqslant C N^{\gamma_{+}-\gamma_{-}+\epsilon}\|u\|_{\gamma, 0, L}
\end{aligned}
$$

for any $\epsilon>0$; here $C$ depends on $\epsilon$.

Remark. Estimate (3.44) cannot be essentially improved. This can be shown using results of Nitsche [17].

(b) Finite elements. For each $0<h \leqslant 1$ let $\Delta^{h}$ be a partition of $[0,2 \pi]$ into subintervals of length less than or equal to $h$. By such a partition we mean a sequence $\left\{x_{j}\right\}_{j=0}^{N}$ satisfying 


$$
\begin{aligned}
& 0 \leqslant x_{0}<x_{1}<\cdots<x_{N-1}<2 \pi \leqslant x_{N}, \\
& x_{N}-x_{0}=2 \pi \text { and } \\
& \max _{j=0, \ldots, N-1}\left(x_{j+1}-x_{j}\right) \leqslant h .
\end{aligned}
$$

We can consider our partition to be extended to all of $(-\infty,+\infty)$ by "periodicity", i.e., we can let $x_{j+N}=x_{j}$ for $j=\ldots,-1,0,1, \ldots$. We assume the family $\left\{\Delta^{h}\right\}_{0<h \leqslant 1}$ is quasi-uniform, i.e., there is a constant $C$ such that $h \leqslant C \min _{j=0}, \ldots, N-1\left(x_{j+1}-x_{j}\right)$ for all $0<h \leqslant 1$.

For $r$ and $q$ integers satisfying $0 \leqslant q, 2 q+1 \leqslant r$ we let

$$
S^{h}=S^{h}(r, g)=\left\{f: f \in C^{q}(R), f \text { is } 2 \pi\right. \text {-periodic, }
$$

$f$ is a polynomial of degree $r$ on each subinterval of $\left.\Delta^{h}\right\}$.

The family $\left\{S_{h}\right\}_{0<h \leqslant 1}$ is a $(t, k)$-regular system with $k=q+1$ and $t=r+1$ (cf. Babuška-Aziz [2, pp. 83,84$])$, i.e., it satisfies the following approximability properties:

(1) $S^{h} \subset H^{k}$ for all $0<h \leqslant 1$,

(2) for $0 \leqslant l, 0 \leqslant s \leqslant \min (l, k), l$ and $s$ constant, there is a constant $C=C(l, s)$ such that for each $u \in H^{l}$ there is a $\phi=\phi(u, h) \in S^{h}$ such that

$$
\|u-\phi\|_{s} \leqslant C h^{\mu}\|u\|_{l}, \quad 0 \leqslant s \leqslant \min (l, k),
$$

where $\mu=\min (t-s, l-s)$.

In fact, we may take $\phi$ to be $p_{h} u$ where $p_{h} u$ is the $H^{0}$-orthogonal projection of $u$ onto $S^{h}$. In addition, the following inverse property holds: for $0 \leqslant \alpha \leqslant \beta \leqslant q+1, \alpha$ and $\beta$ constant, there is a constant $C=C(\alpha, \beta)$ such that

$$
\|x\|_{\beta} \leqslant C h^{\alpha-\beta}\|x\|_{\alpha}
$$

for all $\chi \in S^{h}$ and all $0 \leqslant h \leqslant 1$.

We next present a result which establishes the approximability property of $\left\{S^{h}\right\}_{0<k \leqslant 1}$ with respect to variable order norms. In the proof of this result we need a lemma first proved by Douglas, Dupont and Wahlbin [8]. (More specifically, this lemma is a consequence of inequality (4.7) in [8].) Compare also de Boor [4] and Nitsche and Schatz [18], [19]. For the sake of completeness we present a proof of this lemma that is naturally related to the ideas of our paper.

Lemma. Let $0<\delta<\xi<\pi$ and $0<\epsilon<1$, and set $I_{\xi}=\{x: \xi<x<2 \pi-\xi\}$. Then, there are constants $C$ and $\eta>0$ such that

$$
\left(\int_{I_{\xi}}\left|\frac{d^{j} w_{h}}{d x^{j}}\right|^{2} d x\right)^{1 / 2} \leqslant C e^{-\eta h^{-\epsilon}} h^{-j}\|w\|_{0}, \quad j=0,1, \ldots, q+1,
$$

for all $w \in H^{0}$ with $(\operatorname{supp} w) \cap[-\pi, \pi] \subset(-\delta, \delta)$ and all $h$, where $w_{h}=p_{h} w$ is the $H^{0}$-orthogonal projection of $w$ on $S^{h}$. C and $\eta$ depend on $\delta$, $\xi$ and $\delta$ but not on $w$ and $h$.

Proof. We divide the proof into several parts.

(1) Let $\psi_{h}(x)$ be a family of functions with the properties:

(1) $\psi_{h} \in E_{R}$, 
(2) $0<\psi_{h}(x), \psi_{h}((\delta+\xi) / 2)=1, \psi_{h}(x)$ is decreasing for $0 \leqslant x \leqslant \pi$,

(3) $\psi_{h}(-x)=\psi_{n}(x)$,

(4) $\psi_{h}(x)=\cosh h^{-\epsilon}(\pi-x) / \cosh h^{-\epsilon}(\pi-(\delta+\xi) / 2),(\delta+\xi) / 2 \leqslant x \leqslant \pi$,

(5) $\left|\psi_{h}^{(p)}(x)\right| \leqslant C_{p}\left(h^{-\epsilon}\right)^{p} \psi_{h}(x)$ for all $p, x$ and $h$, where $C_{p}$ is independent of $x$ and $h$.

Such a family can be constructed as follows. Let

$$
f_{h}(x)=\cosh h^{-\epsilon}(\pi-x) / \cosh h^{-\epsilon}\left(\pi-\frac{\delta+\xi}{2}\right)
$$

and let $\mu \in C^{\infty}(-\infty, \infty)$ satisfy $\mu(x)=0$ for $x<-(\delta+\xi) / 2$ and $\mu(x)=1$ for $x \geqslant 0$.

Now let $\psi_{h}(x)$ be the $C^{\infty}$, even, $2 \pi$-periodic function defined by

$$
\psi_{h}(x)=\int_{\pi}^{x} \mu\left(h^{-\epsilon}\left(t-\frac{\delta+\xi}{2}\right)\right) f_{h}^{\prime}(t) d t+f_{h}(\pi), \quad 0 \leqslant x \leqslant \pi .
$$

It is easily seen that this family has the above properties.

As a consequence of property (5) we have

$$
\left|\left(\psi_{h}^{-1}\right)^{(p)}(x)\right| \leqslant C_{p}\left(h^{-\epsilon}\right)^{p} \psi_{h}^{-1}(x)
$$

and

$$
\max _{x \in I_{j}} \psi_{h}(x) / \min _{x \in I_{j}} \psi_{h}(x) \leqslant C
$$

for $j=1, \ldots, N$ and all $h$, where $I_{j}=\left[x_{j-1}, x_{j}\right], j=1, \ldots, N$, are the subintervals of the partition $\Delta^{h}$.

(2) Let $H_{\psi_{h}}^{0}$ and $H_{\psi_{h}^{-1}}^{0}$ denote the space $S^{h}$ equipped with the norms

$$
\|u\|_{\psi_{h}}^{2}=\int_{0}^{2 \pi}|u|^{2} \psi_{h} d x
$$

and

$$
\|u\|_{\psi_{h}^{-1}}^{2}=\int_{0}^{2 \pi}|u|^{2} \psi_{h}^{-1} d x
$$

respectively. Consider the sesquilinear form $G(u, v)=\int_{0}^{2 \pi} u \bar{v} d x$ on $S^{h} \times S^{h}$. Clearly

$$
G(u, v) \leqslant\|u\|_{\psi_{h}^{-1}}\|v\|_{\psi_{h}}
$$

for $u, v \in S^{h}$.

Let $u \in S^{h}$ and set $v=u \psi_{h}^{-1}$. Now let $v_{h}$ be an $S^{h}$-interpolant of $v$ (defined on $I_{j}$ by setting $v_{h}^{(l)}(x)=v^{(l)}(x), l=0,1, \ldots, q, x=x_{j-1}, x_{j}$, and $v_{h}(x)=v(x)$ for any $r-(2 q+1)$ uniformly spaced values of $x$ in $\left.\left(x_{j-1}, x_{j}\right)\right)$. Then we have the standard estimate

$$
\left\|v-v_{h}\right\|_{0, I_{j}} \leqslant C h^{r+1}\left|v^{(r+1)}\right|_{0, I_{j}}, \quad j=1, \ldots, N .
$$

Now, recalling that $u$ is a polynomial of degree $r$ on $I_{j}$, we have

$$
v^{(r+1)}=\sum_{i=0}^{r}\left(\begin{array}{c}
r+1 \\
i
\end{array}\right) u^{(i)}\left(\psi_{h}^{-1}\right)^{(r+1-i)} .
$$

Combining (3.50), (3.51) and (3.47) and using the inverse estimate $\left|u^{(i)}\right|_{0, I_{j}} \leqslant$ 
$C h^{-i}|u|_{0, I_{j}}$ we have, after a simple computation,

Thus

$$
\begin{aligned}
\left\|v-v_{j}\right\|_{0, I_{j}} & \leqslant C h^{r+1} \sum_{i=0}^{r}\left|u^{(i)}\left(\psi_{h}^{-1}\right)^{(r+1-i)}\right|_{0, I_{j}} \\
& \leqslant C h^{1-\epsilon} \max _{I_{j}} \psi_{h}^{-1}|u|_{0, I_{j}}
\end{aligned}
$$

$$
\begin{aligned}
\left\|v-v_{h}\right\|_{\psi_{h}}^{2} & \leqslant C \sum_{j=1}^{N} \max _{I_{j}} \psi_{h} \max _{I_{j}} \psi_{h}^{-2} h^{2(1-\epsilon)}|u|_{0, I_{j}}^{2} \\
& \leqslant C h^{2(1-\epsilon)} \sum_{j=1}^{N} \frac{\max _{I_{j}} \psi_{h} \max _{I_{j}} \psi_{h}^{-2}}{\min _{I_{j}} \psi_{h}^{-1}} \int_{I_{j}}|u|^{2} \psi_{h}^{-1} d x .
\end{aligned}
$$

Now, from (3.48) we have

$$
\frac{\max _{I_{j}} \psi_{h} \max _{I_{j}} \psi_{h}^{-2}}{\min _{I_{j}} \psi_{h}^{-1}}=\frac{\max _{I_{j}} \psi_{h}^{2}}{\min _{I_{j}} \psi_{h}^{2}} \leqslant C
$$

for all $j$ and $h$. Thus we have

$$
\left\|v-v_{h}\right\|_{\psi_{h}}^{2} \leqslant C h^{2(1-\epsilon)} \sum_{j=1}^{N} \int_{I_{j}}|u|^{2} \psi_{h}^{-1} d x=C h^{2(1-\epsilon)}\|u\|_{\psi_{h}^{-1}}^{2} .
$$

Using this and recalling that $v=u \psi_{h}^{-1}$, we get

and

$$
\left|G\left(u, v_{h}\right)\right| \geqslant|G(u, v)|-\left|G\left(u, v-v_{h}\right)\right| \geqslant\|u\|_{\psi_{h}^{-1}}^{2}\left(1-C h^{1-\epsilon}\right)
$$

$$
\left\|v_{h}\right\|_{\psi_{h}} \leqslant\|v\|_{\psi_{h}}+\left\|v-v_{h}\right\|_{\psi_{h}} \leqslant C\|u\|_{\psi_{h}^{-1}}
$$

from which we obtain

$$
\sup _{\substack{v \in S^{h} \\\|v\|_{\psi_{h}}=1}}|G(u, v)| \geqslant C_{1}\|u\|_{\psi_{h}^{-1}}
$$

for all $h$ and all $u \in S^{h}$, where $C_{1}>0$ is independent of $u$ and $h$.

(3) Let $w \in H^{0}$ with (supp $\left.w\right) \cap[-\pi, \pi] \subset(-\delta, \delta) . w_{h}=p_{h} w$ satisfies

$$
\begin{aligned}
w_{h} & \in S^{h}, \\
G\left(w_{h}, \phi\right) & =G(w, \phi), \quad \phi \in S^{h} .
\end{aligned}
$$

Thus, using (3.49) and (3.52) and recalling that $\psi_{h}(x) \geqslant 1$ for $|x| \leqslant \delta$, we have

$$
C_{1}\left\|w_{h}\right\|_{\psi_{h}^{-1}} \leqslant \sup _{\substack{\phi \in S^{h} \\\|\phi\|_{\psi_{h}}=1}}\left|G\left(w_{h}, \phi\right)\right|=\sup _{\substack{\phi \in S^{h} \\\|\phi\|_{\psi_{h}}=1}}|G(w, \phi)| \leqslant\|w\|_{\psi_{h}^{-1}} \leqslant\|w\|_{0}
$$

(cf. Babuška and Aziz [2, p. 112]). Hence 


$$
\int_{I_{\xi}}\left|w_{h}\right|^{2} d x \leqslant \psi_{h}(\xi) \int_{I_{\xi}}\left|w_{h}\right|^{2} \psi_{h}^{-1} d x \leqslant \frac{\psi_{h}(\xi)}{C_{1}^{2}}\|w\|_{0}^{2} .
$$

Now, using property (4), it is easily seen that

$$
\psi_{h}(\xi) \leqslant 2 e^{-h^{-\epsilon}(\xi-\delta) / 2}
$$

Combining (3.53) and (3.54), we have

$$
\left(\int_{I_{\xi}}\left|w_{h}\right|^{2} d x\right)^{1 / 2} \leqslant C e^{-\eta h^{-\epsilon}}\|w\|_{0},
$$

where $C$ and $\eta>0$ depend on $\delta$, $\xi$ and $\epsilon$ but are independent of $w$ and $h$. Now the desired result follows from the inverse property of the family $\left\{S^{h}\right\}$.

THEOREM 7. Suppose $\gamma(x) \in E_{R}$ and $\mu$ is a constant which satisfy $0 \leqslant \gamma(x)<$ $q+1, \gamma_{+}-\gamma_{-}<1,0 \leqslant \mu, \gamma_{+}+\mu \leqslant r+1$. Then for each $\epsilon>0$ there is a constant $C(\epsilon)$ such that

$$
\inf _{x \in S^{h}}\|u-\chi\|_{\gamma, 0, L} \leqslant C(\epsilon) h^{\mu-\epsilon}\|u\|_{\gamma, \mu, L}
$$

for all $u \in H^{\gamma, \mu, L}$ and all $0<h \leqslant 1$.

Proof. Given $\gamma$ and $\epsilon$ we can choose $\theta$ and $\vec{p}_{\theta}$ such that

$$
\gamma \sim \vec{p}_{\theta}
$$

and

$$
p_{\theta, j-1}^{+}-p_{\theta, j}^{-}<\epsilon, \quad p_{\theta, j}^{+}-p_{\theta, j}^{-}<\epsilon, \quad p_{\theta, j+1}^{+}-p_{\theta, j}^{-}<\epsilon, \quad p_{\theta, j}^{+} \leqslant q+1,
$$

$$
p_{\theta, j}^{+}+(\mu-\epsilon) \leqslant r+1, \quad j=1, \ldots, M .
$$

Let $E_{h}(u)=u-p_{h} u$, where $p_{h} u$ is the $H^{0}$-orthogonal projection on $S^{h}$. Using Theorem 5 in Chapter 2, we see that

$$
\begin{aligned}
\left\|E_{h}(u)\right\|_{\gamma, 0, L} & \leqslant C\left\{\sum_{l=1}^{M}\left\|E_{n}(u) x_{\theta, l}\right\|_{p_{\theta, l}^{+}}+\left\|E_{h}(u)\right\|_{-L}\right\} \\
& \leqslant C\left\{\sum_{j} \sum_{l}\left\|E_{h}\left(u \chi_{\theta, j}\right) \chi_{\theta, l}\right\|_{p_{\theta, l}^{+}}+\left\|E_{h}(u)\right\|_{-L}\right\} .
\end{aligned}
$$

Consider $E_{h}\left(u \chi_{\theta, j}\right) \chi_{\theta, l}$ and suppose first that $l \neq j-1, j+1$. (If $j=1$, we suppose $l \neq M, 1,2$; and if $j=M$, we suppose $l \neq m-1, M, 1$; for simplicity of notation we will in any case indicate this condition by writing $|l-j| \geqslant 2$.) Using the lemma, we have

$$
\begin{aligned}
\left\|E_{h}\left(u \chi_{\theta, j}\right) \chi_{\theta, l}\right\|_{p_{\theta, l}^{+}} & \leqslant\left(\sum_{i=0}^{q+1} \int_{|x-3 \theta l / 2| \leqslant \theta}\left|\frac{d^{i}}{d x^{i}} E_{n}\left(u \chi_{\theta, j}\right)\right|^{2} d x\right)^{1 / 2} \\
& \leqslant C h^{\mu}\left\|u \chi_{\theta, j}\right\|_{0} \leqslant C h^{\mu}\|u\|_{0} .
\end{aligned}
$$


Now suppose $|l-j| \leqslant 1$. Using the approximability property (3.45) we have

$$
\left\|E_{h}\left(u \chi_{\theta, j}\right) \chi_{\theta, l}\right\|_{p_{\theta, l}^{+}} \leqslant C\left\|E_{h}\left(u \chi_{\theta, j}\right)\right\|_{p_{\theta, l}^{+}} \leqslant C h^{\mu-\epsilon}\left\|u \chi_{\theta, j^{\prime}}\right\|_{p_{\theta, l}^{+}(\mu-\epsilon)}
$$

provided $0<\epsilon \leqslant \mu$ since $p_{\theta, l}^{+}+(\mu-\epsilon) \leqslant r+1$. Using the inverse property (3.46) together with (3.45) we have

$$
\left\|E_{h}\left(u \chi_{\theta, j}\right)\right\|_{p_{\theta, l}^{+}} \leqslant C h^{-\epsilon}\left\|E_{h}\left(u \chi_{\theta, j}\right)\right\|_{p_{\theta, l^{+}}} \leqslant C h^{-\epsilon}\left\|u \chi_{\theta, j^{\prime}}\right\|_{p_{\theta, l^{+}}^{+\epsilon}} .
$$

Thus (3.59) holds for $\mu=0$ and $\epsilon>0$. From (3.56) and (3.59) we have

for $|l-j| \leqslant 1$.

$$
\left\|E_{h}\left(u \chi_{\theta, j}\right) \chi_{\theta, l}\right\|_{p_{\theta, l}^{+}} \leqslant C h^{\mu-\epsilon}\left\|u \chi_{\theta, j^{\prime}}\right\|_{p_{\theta, j}+\mu}
$$

Combining (3.58) and (3.60), we get

$$
\begin{aligned}
\sum_{j} \sum_{l} & \left\|E_{h}\left(u \chi_{\theta, j}\right) \chi_{\theta, l}\right\|_{p_{\theta, l}^{+}} \\
& =\sum_{j=1}^{M} \sum_{|l-j| \leqslant 1}\left\|E_{h}\left(u \chi_{\theta, j}\right) \chi_{\theta, l^{\prime}}\right\|_{p_{\theta, l}^{+}}+\sum_{j=1}^{M} \sum_{|l-j|>1}\left\|E_{h}\left(u \chi_{\theta, j}\right) \chi_{\theta, l}\right\|_{p_{\theta, l}^{+}} \\
& \leqslant C h^{\mu-\epsilon} \sum_{j=1}^{M}\left\|u \chi_{\theta, j}\right\|_{p_{\theta, j}+\mu}+C h^{\mu}\|u\|_{0} .
\end{aligned}
$$

Since (3.55) implies $(\gamma(x)+\mu) \sim\left(\vec{p}_{\theta}+\mu\right)$, we see from Theorem 5 in Chapter 2 that

$$
\sum_{j}\left\|u \chi_{\theta, j^{\prime}}\right\|_{p_{\theta, j}+\mu} \leqslant C\|u\|_{\gamma, \mu, L}
$$

From (3.45) and Theorem 3 in Chapter 2 we have

$$
\left\|E_{h}(u)\right\|_{-L} \leqslant\left\|E_{h}(u)\right\|_{0} \leqslant C h^{\mu}\|u\|_{\mu} \leqslant C h^{\mu}\|u\|_{\gamma, \mu, L} .
$$

Finally, combining (3.57), (3.61) and (3.62) and a further application of Theorem 3 in Chapter 2, we obtain

$$
\left\|u-p_{h} u\right\|_{\gamma, 0, L} \leqslant C(\epsilon) h^{\mu-\epsilon}\|u\|_{\gamma, \mu, L} .
$$

We note that for $0<\mu$ this result holds for $0<\epsilon \leqslant \mu$, and for $\mu=0$ it holds for $\epsilon>0$. This completes the proof of Theorem 7 .

The next theorem is used to verify assumption (3.11) for finite element spaces.

THEOREM 8. Suppose $\gamma \in E_{R}$ with $0<\gamma(x)<q+1$ and $\gamma_{+}-\gamma_{-}<1$. Then for any $\epsilon>0$ there is a constant $C(\epsilon)$ such that

$$
\left\|p_{h} u\right\|_{\gamma(x), 0, L} \leqslant C(\epsilon) h^{-\epsilon}\|u\|_{\gamma(x), 0, L}
$$

for all $u \in H^{\gamma(x), 0, L}$, where $p_{h} u$ is the $H^{0}$-orthogonal projection of $u$ onto $S^{h}$.

Proof. Let $\epsilon>0$. Using Theorem 7 with $\mu=0$ we have

$$
\begin{aligned}
\left\|p_{h} u\right\|_{\gamma, 0, L} & \leqslant\left\|p_{h} u-u\right\|_{\gamma, 0, L}+\|u\|_{\gamma, 0, L} \\
& \leqslant C h^{-\epsilon}\|u\|_{\gamma, 0, L}+\|u\|_{\gamma, 0, L} \leqslant C h^{-\epsilon}\|u\|_{\gamma, 0, L} .
\end{aligned}
$$

If $\gamma$ is constant we note that a direct application of (3.45) yields this result with $\epsilon=0$. 
For $r$ and $q$ integers satisfying $1 \leqslant q$ and $2 q+1 \leqslant r$ we now define $S_{1}^{h}=$ $S^{h}(r, q)$ and $S_{2}^{h}=S^{h}(r-1, q-1)$. With $k_{1}=q+1, k_{2}=q, t_{1}=r+1$ and $t_{2}=r$, assumptions (3.8) and (3.9) are immediate, (3.10) follows from Theorem 7, and (3.11) with $\delta=\epsilon$ for any positive $\epsilon$ follows from Theorem 8. Finally, we observe that if $\gamma$ is constant, then (3.10) and (3.11) with $\epsilon=0$ are consequences of the standard approximability property $(3.45)$.

3.4. Error Estimates for the Eigenvalue Problem. In this section we apply the previously developed theory to estimate the errors which arise when the eigenvalues of the problem (1.7)-(1.10) (which is equivalent to (1.1)-(1.3) and to (1.15)) are approximated by the Ritz-Galerkin method based on the sesquilinear forms (1.20) and (1.21) (cf. (1.15) and (3.4)). We assume throughout this section that $\tau \in H^{\phi, 0, L}$ and $\xi, \rho \in$ $H^{\psi, 0, L}$ where $\phi, \psi \in E_{R}, 0<\phi(x), \psi(x)$ and $\phi_{+}-\phi_{-}<1, \psi_{+}-\psi_{-}<1$. As a preliminary step, we study the regularity of the eigenfunctions of (1.15).

THEOREM 9. Suppose $\lambda_{0}$ is an eigenvalue of (1.15) with $A$ and $B$ given by (1.20) and (1.21), and let $\left(u_{0}, \sigma_{0}\right)$ be a corresponding eigenfunction. Then $u_{0} \in H^{\omega_{1}, 0, L}$ and $\sigma_{0} \in H^{\omega_{2}, 0, L}$, where $\omega_{1}$ and $\omega_{2}$ are any functions in $E_{R}$ satisfying $\omega_{i,+}-\omega_{i,-}<$ $1, i=1,2$, and

$$
0 \leqslant \omega_{1}(x)<\min (\phi(x)+1, \psi(x)+2), \quad 0 \leqslant \omega_{2}(x)<\min (\phi(x)+2, \psi(x)+1) .
$$

Proof. It is immediate that

$$
u_{\mathrm{o}}^{\prime}-\tau \sigma_{0}=0, \quad \sigma_{0}^{\prime}-\xi u_{0}+\lambda_{0} \rho u_{0}=0
$$

and $u_{0}, \sigma_{0} \in H^{1}$. Using Theorem 10 of Chapter 2 , we get $u_{0}^{\prime} \in H^{\eta_{1}, 0, L}$ where $\eta_{1}$ is any function in $E_{R}$ satisfying $\eta_{1,+}-\eta_{1,-}<1$ and $0 \leqslant \eta_{1}(x)<\min (1, \phi(x))$. Now, using Theorems 2, 3 and 4 in Chapter 2 we see that $u_{0} \in H^{\eta_{2}, 0, L}$ for any $\eta_{2} \in E_{R}$ such that $\eta_{2,+}-\eta_{2,-}<1$ and $0 \leqslant \eta_{2}(x)<\min (2, \phi(x)+1)$. Analogously we find that $\sigma_{0} \in H^{\eta_{3}, 0, L}$ for any $\eta_{3} \in E_{R}$ with $\eta_{3,+}-\eta_{3,-}<1$ and $0 \leqslant \eta_{3}(x)<$ $\min (3, \phi(x)+2, \psi(x)+1)$. Proceeding in this way we obtain the desired result.

Now we analyze separately the two special choices introduced in Chapter 3, namely trigonometric polynomials and finite elements.

(a) Trigonometric polynomials. First we prove assumption (3.3) for this particular choice.

THEOREM 10. Assumption (3.3) is satisfied for trigonometric polynomials provided that

$$
\begin{gathered}
\beta_{+}<2 \beta_{-}, \\
2 \beta_{+}-\beta_{-}<1 \quad\left(\text { or } \alpha_{+}<2 \alpha_{-}\right) .
\end{gathered}
$$

Proof. We must show that

$$
\left\|T_{N}-T\right\| \underset{N \rightarrow \infty}{\longrightarrow} 0
$$

where $T$ and $T_{N}$ are defined in Sections 3.1 and 3.3, respectively. Let $(u, \sigma) \in H=$ $H^{\alpha, 0, L} \times H^{\beta, 0, L}$ and set $T(u, \sigma)=(\widetilde{u}, \widetilde{\sigma})$. Then $\widetilde{u}, \widetilde{\sigma}$ satisfy

$$
\tilde{u}^{\prime}-\tau \widetilde{\sigma}=0
$$


and

$$
\widetilde{\sigma}^{\prime}-\xi \tilde{u}+\rho u=0 .
$$

Multiplying (4.4) by $\tilde{u}$, integrating by parts, and using (4.3) we obtain

from which we get

$$
\int_{0}^{2 \pi}\left(\left|\tilde{u}^{\prime}\right|^{2} / \tau+\xi|\tilde{u}|^{2}\right) d x=\int_{0}^{2 \pi} \rho u \tilde{u} d x
$$

$$
\left\|\tilde{u}^{\prime}\right\|_{0}^{2} \leqslant C\|u\|_{0}\|\tilde{u}\|_{0}
$$

and

$$
\|\widetilde{u}\|_{0} \leqslant C\|u\|_{0}
$$

Now from (4.3)-(4.6) we see that

$$
\|\widetilde{\sigma}\|_{1} \leqslant C\|u\|_{0} \leqslant C\|(u, \sigma)\|_{H}
$$

and

$$
\|\tilde{u}\|_{1} \leqslant C\|u\|_{0} \leqslant C\|(u, \sigma)\|_{H} .
$$

Using (4.1) and the fact that $\beta_{+}-\beta_{-}<1$, we can choose $\delta_{1}>0$ such that

$$
3 \delta_{1}<\beta_{-}-\left(\beta_{+}-\beta_{-}\right), \quad\left(\beta_{+}-\beta_{-}\right)+3 \delta_{1} \leqslant 1 .
$$

Then from Theorem 5, Theorem 7 in Chapter 2, and (4.8) we have

$$
\inf _{\chi \in S^{N}}\|\tilde{u}-\chi\|_{\alpha, 0, L} \leqslant \frac{C}{N^{\left(\beta_{+}-\beta_{-}\right)+2 \delta_{1}}}\|\tilde{u}\|_{\alpha_{,}\left(\beta_{+}-\beta_{-}\right)+3 \delta_{1}, L}
$$

$$
\leqslant \frac{C}{N^{\left(\beta_{+}-\beta_{-}\right)+2 \delta_{1}}}\|\tilde{u}\|_{1} \leqslant \frac{C}{N^{\left(\beta_{+}-\beta_{-}\right)+2 \delta_{1}}}\|(u, \sigma)\|_{H^{*}}
$$

Using (4.2), we can choose $\delta_{2}>0$ so that

$$
3 \delta_{2}<\left(1-\beta_{+}\right)-\left(\beta_{+}-\beta_{-}\right), \quad\left(\beta_{+}-\beta_{-}\right)+3 \delta_{2} \leqslant 1 .
$$

From Theorem 5, Theorem 7 in Chapter 2, and (4.7) we get

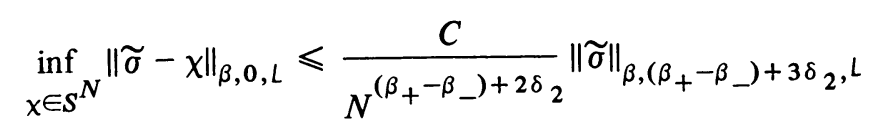

$$
\leqslant \frac{C}{N^{\left(\beta_{+}-\beta_{-}\right)+2 \delta_{2}}}\|(u, \sigma)\|_{H}
$$

Now it follows (see Babuška-Aziz [2, pp. 112, 187]) from Theorem 4, Theorem 6, (4.9) and (4.10) that

$$
\left\|\left(T-T_{h}\right)(u, \sigma)\right\|_{H} \leqslant C N^{-\delta_{3}}\|(u, \sigma)\|_{H},
$$

where $\delta_{3}=\min \left(\delta_{1}, \delta_{2}\right)>0$. This completes the proof.

All of the assumptions (3.1)-(3.3) have now been verified for approximation by trigonometric polynomials. Let $\lambda_{0}$ be an eigenvalue of (1.15) with the sesquilinear forms (1.20) and (1.21) and let $\lambda_{0}^{N}$ be its approximation based on trigonometric poly- 
nomials of degree $N$. We may now apply Theorem 3 to estimate $\lambda_{0}-\lambda_{0}^{N}$.

THEOREM 11. Suppose $\tau \in H^{\phi, 0, L}$ and $\xi, \rho \in H^{\psi, 0, L}$, where $\phi, \psi \in E_{R}, 0<$ $\phi(x), \psi(x)$ and $\phi_{+}-\phi_{-}<1, \psi_{+}-\psi_{-}<1$. Suppose $\beta \in E_{R}$ satisfies $0<\beta(x)<1$, (4.1) and (4.2). Then for every $\epsilon>0$ there is a constant $C(\epsilon)$ such that

$$
\left|\lambda_{0}-\lambda_{0}^{N}\right| \leqslant C(\epsilon) N^{-2 \eta+\epsilon}
$$

where

$$
\begin{aligned}
\eta_{1} & =\min _{0 \leqslant x \leqslant 2 \pi} \min (\phi(x)+\beta(x), \psi(x)+1+\beta(x)), \\
\eta_{2} & =\min _{0 \leqslant x \leqslant 2 \pi} \min (\phi(x)+1+\alpha(x), \psi(x)+\alpha(x)), \\
\eta & =\min \left(\eta_{1}, \eta_{2}\right)-\left(\beta_{+}-\beta_{-}\right) / 2 .
\end{aligned}
$$

Proof. This result follows immediately from Theorems 3 (estimate (3.6) together with equation (3.5)), 5, 6, and 9 .

The estimate (4.11) depends on $\beta(x)$. For $\phi(x)$ and $\psi(x)$ given we now consider the problem of the optimal choice for $\beta(x)$. We remark that the approximations $\lambda_{0}^{N}$ do not depend on $\beta(x)$; the choice of $\beta(x)$ determines only the error estimate that we can establish with Theorem 3. The next theorem shows that the optimal choice for $\beta(x)$ is a constant function.

THEOREM 12. Suppose $\beta(x) \in E_{R}, 0<\beta(x)<1$. Let $\beta_{0}$ be the constant function defined by

$$
\beta_{0}= \begin{cases}\kappa & \text { if } 0 \leqslant \kappa \equiv\left(\psi_{-}-\phi_{-}+1\right) / 2 \leqslant 1 \\ 0 & \text { if } \kappa<0 \\ 1 & \text { if } \kappa>1\end{cases}
$$

and let $\eta^{0}$ be determined from $\beta_{0}$ according to (4.12)-(4.14), i.e., let

$$
\psi^{0}= \begin{cases}\psi_{-}+1 & \text { if } \kappa<0, \\ \left(\psi_{-}+\phi_{-}+1\right) / 2 & \text { if } 0 \leqslant \kappa \leqslant 1, \\ \phi_{-}+1 & \text { if } \kappa>1 .\end{cases}
$$

Then $\eta^{0} \geqslant \eta$, where $\eta$ is determined from $\beta(x)$ according to (4.12)-(4.14).

Proof. From (4.12) and (4.13) we have

$$
\eta_{1} \leqslant \phi_{-}+\beta_{+} \text {and } \eta_{2} \leqslant \psi_{-}+1-\beta_{-} \text {. }
$$

From these inequalities it is immediate that

$$
\begin{aligned}
\eta & \leqslant \min \left(\phi_{-}+\beta_{+}-\frac{\beta_{+}-\beta_{-}}{2}, \psi_{-}+1-\beta_{+}-\frac{\beta_{+}-\beta_{-}}{2}\right) \\
& =\min \left(\phi_{-}+\frac{\beta_{+}-\beta_{-}}{2}, \psi_{-}+1-\frac{\beta_{+}-\beta_{-}}{2}\right) .
\end{aligned}
$$

If $\kappa<0$, then $\psi_{-}+1 \leqslant \phi_{-}$and hence 


$$
\eta \leqslant \psi_{-}+1-\left(\beta_{+}-\beta_{-}\right) / 2 \leqslant \psi_{-}+1=\eta^{0},
$$

and if $\kappa>1$, then $\phi_{-}+1<\psi_{-}$and hence,

$$
\begin{aligned}
\eta & \leqslant \min \left(\phi_{-}+\frac{\beta_{+}+\beta_{-}}{2}, \psi_{-}+1-\frac{\beta_{+}+\beta_{-}}{2}\right) \\
& =\min \left(\phi_{-}+1-\frac{\beta_{+}+\beta_{-}}{2}, \psi_{-}+\frac{\alpha_{+}+\alpha_{-}}{2}\right) \leqslant \phi_{-}+1=\eta^{0} .
\end{aligned}
$$

In the case $0 \leqslant \kappa \leqslant 1$ we have

$$
\begin{aligned}
\eta & \leqslant \min \left(\phi_{-}+\frac{\beta_{+}-\beta_{-}}{2}, \psi_{-}+1-\frac{\beta_{+}-\beta_{-}}{2}\right) \\
& \leqslant \max _{\nu \geqslant 0} \min \left(\phi_{-}+\nu, \psi_{-}+1-\nu\right)=\frac{\phi_{-}+\psi_{-}+1}{2}=\eta^{0} .
\end{aligned}
$$

Thus we see that for the case of approximation by trigonometric polynomials the use of variable order spaces does not lead to improved error estimates. Using Theorem 12 , we readily see that the optimal estimate obtainable from Theorem 11 is given by

$$
\eta= \begin{cases}\psi_{-}+1 & \text { if } \kappa<0, \\ \left(\phi_{-}+\psi_{-}+1\right) / 2 & \text { if } 0 \leqslant \kappa \leqslant 1, \\ \phi_{-}+1 & \text { if } 0 \leqslant \kappa \leqslant 1 .\end{cases}
$$

(b) Finite elements. Here we let

$$
S_{1}^{h}=S^{h}(r, q) \text {. and } S_{2}^{h}=S^{h}(r-1, q-1) \text {, }
$$

where $r$ and $q$ are integers satisfying $1 \leqslant q$ and $2 q+1 \leqslant r$. Our next theorem establishes assumption (3.3) for finite elements.

TheOREM 13. Assumption (3.3) is satisfied for finite elements.

Proof. The proof is similar to that of Theorem 10 . We easily see that it is sufficient to show that

$$
\begin{aligned}
& \inf _{x \in S_{1}^{h}}\|\tilde{u}-\chi\|_{\alpha, 0, L} \leqslant C h^{\delta}\|(u, \sigma)\|_{H}, \\
& \inf _{x \in S_{2}^{h}}\|\tilde{\sigma}-\chi\|_{\beta, 0, L} \leqslant C h^{\delta}\|(u, \sigma)\|_{H}
\end{aligned}
$$

with $\delta>0$ independent of $h$. (4.15) and (4.16) follow immediately from Theorem 7, (4.7) and (4.8).

All of the assumptions (3.1)-(3.3) have been verified for approximation by finite elements. We may now apply Theorem 3 to estimate $\lambda_{0}-\lambda_{0}^{h}$, where $\lambda_{0}$ is an eigenvalue of (1.15) with sesquilinear forms (1.20) and (1.21) and $\lambda_{0}^{h}$ is its approximation based on $M^{h}=S_{1}^{h} \times S_{2}^{h}$.

THEOREM 14. Suppose $\tau \in H^{\phi, 0, L}$ and $\xi, \rho \in H^{\psi, 0, L}$, where $\phi, \psi \in E_{R}, 0<$ $\phi(x), \psi(x)$ and $\phi_{+}-\phi_{-}<1, \psi_{+}-\psi_{-}<1$. Suppose $\beta \in E_{R}$ satisfies $0<\beta(x)<1$. 
Then for every $\epsilon>0$ there is a constant $C(\epsilon)$ such that

$$
\left|\lambda_{0}-\lambda_{0}^{h}\right| \leqslant C(\epsilon) h^{2 \eta-\epsilon}
$$

where

$$
\begin{aligned}
\eta_{1} & =\min _{0 \leqslant x \leqslant 2 \pi} \min (\phi(x)+\beta(x), \psi(x)+1+\beta(x), r+\beta(x)), \\
\eta_{2} & =\min _{0 \leqslant x \leqslant 2 \pi} \min (\phi(x)+1+\alpha(x), \psi(x)+\alpha(x), r-1+\alpha(x)), \\
\eta & =\min \left(\eta_{1}, \eta_{2}\right) .
\end{aligned}
$$

Proof. This theorem follows directly from Theorems 3 (estimate (3.6) together with Eq. (3.5)), 7, 8 and 9.

With regard to Theorems 11 and 14 we remark that we may take $\epsilon=0$ if we are using only constant order spaces.

For $\phi$ and $\psi$ given we now analyze the optimal choice for $\beta(x)$. We again remark that $\beta$ influences the error estimate (4.17) but does not influence the approximation $\lambda_{0}^{h}$. First assume that $r$ is sufficiently large. Define

$$
\begin{aligned}
& I_{1}=\{x: \psi(x)+1<\phi(x)\}, \\
& I_{2}=\{x: \psi(x)-1 \leqslant \phi(x) \leqslant \psi(x)+1\} \text { and } \\
& I_{3}=\{x: \phi(x)<\psi(x)-1\} .
\end{aligned}
$$

We note that since $\phi_{+}-\phi_{-}<1$ and $\psi_{+}-\psi_{-}<1$ at most two of these sets is nonempty. Let

$$
\gamma_{1}(x)=\min (\phi(x)+\beta(x), \psi(x)+1+\beta(x), r+\beta(x))
$$

and

$$
\gamma_{2}(x)=\min (\phi(x)+1+\alpha(x), \psi(x)+\alpha(x), r-1+\alpha(x)) .
$$

Then we see that for $x \in I_{1}$,

$$
\gamma_{1}(x)=\psi(x)+1+\beta(x) \text { and } \gamma_{2}(x)=\psi(x)+1-\beta(x)
$$

while for $x \in I_{3}$,

$$
\gamma_{1}(x)=\phi(x)+\beta(x) \text { and } \gamma_{2}(x)=\phi(x)+2-\beta(x) .
$$

Finally, for $x \in I_{2}$ we have

$$
\gamma_{1}(x)=\phi(x)+\beta(x) \text { and } \gamma_{2}(x)=\psi(x)+1-\beta(x) .
$$

Since $\eta=\min \left(\eta_{1}, \eta_{2}\right)$, we try to choose $\beta(x)$ so that $\eta_{1}=\eta_{2}$. This suggests the choice

$$
\hat{\beta}(x)= \begin{cases}0, & x \in I_{1}, \\ (\psi(x)-\phi(x)+1) / 2, & x \in I_{2}, \\ 1, & x \in I_{3} .\end{cases}
$$

Note that this defines $\hat{\beta}(x)$ as a continuous, but not infinitely differentiable, function. With this choice for $\hat{\beta}(x)$ we see that 


$$
\begin{aligned}
\gamma_{1}(x)=\gamma_{2}(x) & = \begin{cases}\psi(x)+1, & x \in I_{1}, \\
(\psi(x)+\phi(x)+1) / 2, & x \in I_{2}, \\
\phi(x)+1, & x \in I_{3},\end{cases} \\
& \equiv \kappa(x) .
\end{aligned}
$$

Now it is clear that there exists $\beta(x) \in E_{R}$ such that (4.17) holds with

$$
\eta=\min _{0 \leqslant x \leqslant 2 \pi} \kappa(x) \text {. }
$$

It suffices to choose $\beta \in E_{R}$ very close to $\hat{\beta}$.

We note finally that if $r$ is not sufficiently large then the rate of convergence given by (4.17) will have an upper threshold determined by $r$.

3.5. Comparison of Methods. The eigenvalues of (1.1)-(1.3) can be approximated by the Ritz-Galerkin method based on (1.15) with the choice of sesquilinear forms $A$ and $B$ given by (1.16) and (1.17), (1.18) and (1.19), or (1.20) and (1.21). If the forms are given by (1.16) and (1.17), we have the standard Ritz method. We will refer to the method associated with the choice (1.18) and (1.19) as the inverted Ritz method. In this section we make a comparison of these methods.

Suppose $\tau(x) \in H^{\eta}, 0<\eta<1$, and $\xi(x), \rho(x) \in H^{1}$. Then $u_{0} \in H^{1+\eta}$ and the standard Ritz method leads to the rate of convergence $N^{-2 \eta}$ for approximation by trigonometric polynomials and $h^{2 \eta}$ when finite elements are used. These estimates cannot, in general, be improved. This follows from a result giving a lower bound for the eigenvalue error in Ritz approximation (see Kolata [11] and Chatelin [7]). Next we apply the Ritz-Galerkin method based on the forms (1.20) and (1.21). It follows from Theorems 11 and 12 and the discussion following Theorem 12 that the rate of convergence is at least $N^{-(2+\eta)}$ for trigonometric polynomials and $h^{2+\eta}$ for finite elements. Finally we observe that the inverted Ritz method leads to rates of convergence of $N^{-2}$ and $h^{2}$ for trigonometric polynomials and finite elements, respectively.

Suppose next that $\tau \in H^{1}$ and $\xi, \rho \in H^{\eta}, 0<\eta<1$. Here we get rates of convergence $N^{-2}$ and $h^{2}$ for the Ritz method, and $N^{-2 \eta}$ and $h^{2 \eta}$ for the inverted Ritz method. For the Ritz-Galerkin method based on the forms (1.20) and (1.21) we get $N^{-(2+\eta)}$ and $h^{2+\eta}$.

These examples show that if $\tau$ is rough and $\xi$ and $\rho$ are smooth, then the inverted Ritz method leads to a higher rate of convergence than does the Ritz method. Similarly, if $\tau$ is rough and $\xi$ and $\rho$ are smooth, then the Ritz method leads to a higher rate of convergence than does the inverted Ritz method. Further we note that for both of these examples the method based on the forms (1.20) and (1.21) leads to a higher rate than does either the Ritz or inverted Ritz method.

We now analyze thoroughly one very special class of examples. Let $\tau(x), \xi(x)$ and $\rho_{t}(x)$ be defined by

$$
\tau(x)= \begin{cases}1, & |x| \leqslant \pi / 2, \\ 1 / 2, & -\pi \leqslant x<\pi / 2 \text { or } \pi / 2 \leqslant x<\pi,\end{cases}
$$




$$
\begin{aligned}
& \xi(x)=1, \\
& \rho_{t}(x)= \begin{cases}1, & |x-t| \leqslant \pi / 2, \\
1 / 2, & -\pi \leqslant x-t \leqslant-\pi / 2 \text { or } \pi / 2 \leqslant x-t<\pi,\end{cases}
\end{aligned}
$$

where $t$ is a parameter with $|t|<1 / 2$. By applying Theorem 11 in Chapter 2 we see that $\tau \in H^{\phi, 0, L}$ for any $\phi \in E_{R}$ with $\phi_{+}-\phi_{-}<1$ and $\phi( \pm \pi / 2)<1 / 2$. Likewise $\rho_{t} \in$ $H^{\psi} t^{(x), 0, L}$ where $\psi_{t}(x)=\phi(x-t)$.

Consider the case of trigonometric approximation. Using Theorem 9 we see that the standard Ritz method yields the rate $N^{-1+\epsilon}$. From Theorem 12 we see that the rate for the Ritz-Galerkin method based on the forms (1.20) and (1.21) is $N^{-2+\epsilon}$. We note that both of these estimates are independent of the parameter $t$.

Now suppose we are using finite elements and suppose $r$ is sufficiently large. For the standard Ritz method we get $\left|\lambda_{0}-\lambda_{0}^{h}\right| \leqslant C h^{1-\epsilon}$. This estimate does not depend on $t$. Finally we consider the Ritz-Galerkin method based on the forms (1.20) and (1.21). Referring to Theorem 14 and the subsequent discussion we see that $I_{2}=R$ and, thus, $\kappa(x)=\left(\psi_{t}(x)+\phi(x)+1\right) / 2$. Clearly $\phi$ can be chosen so that

$$
\min _{0 \leqslant x \leqslant 2 \pi} \kappa(x)= \begin{cases}1+\phi(1 / 2), & t \neq 0, \\ 1 / 2+\phi(1 / 2), & t=0\end{cases}
$$

Thus we have

$$
\left|\lambda_{, 0}-\lambda_{0}^{h}\right| \leqslant \begin{cases}C h^{2-\epsilon}, & t=0 \\ C h^{3-\epsilon}, & t \neq 0\end{cases}
$$

We see that the rate of convergence for $t=0$ differs from that for $t \neq 0$. We refer to the case $t=0$ as a case of coinciding singularities. This difference in rates of convergence would not be seen if we were analyzing this method with constant order spaces. Then we would obtain $h^{2-\epsilon}$ in both cases.

Finally we remark that problems with rough coefficients arise in many applications. As an example we mention composite material problems (see e.g., [1], [12], $[13],[16])$.

Institute for Physical Science and Technology

Department of Mathematics

University of Maryland

College Park, Maryland 20742

1. I. BABUŠKA, "Homogenization and its applications. Mathematical and computational problems," Numerical Solution of Partial Differential Equations-III (B. Hubbard, Editor), Academic Press, New York, 1976, pp. 89-116.

2. I. BABUŠKA \& A. K. AZIZ, "Survey lectures on the mathematical foundations of the finite element method," The Mathematical Foundations of the Finite Element Method with Applications to Partial Differential Equations (A. K. Aziz, Editor), Academic Press, New York, 1973, pp. 5-359.

3. I. BABUŠKA, J. T. ODEN \& J. K. LEE, "Mixed-hybrid finite element approximations of second order elliptic boundary-value problems," Comput. Methods Appl. Mech. Engrg., v. 11, 1977, pp. 175-206.

4. C. de BOOR, "A bound on the $L_{\infty}$-norm of $L_{2}$-approximation by splines in terms of a global mesh ratio," Math. Comp., v. 30, 1976, pp. 765-771. 
5. J. H. BRAMBLE \& J. E. OSBORN, "Rate of convergence estimates for non-selfadjoint eigenvalue approximations," Math. Comp., v. 27, 1973, pp. 525-549.

6. C. CONUTO, "Eigenvalue approximation by mixed methods," Rev. Française Automat. Informat. Recherche Opérationelle Sér. Rouge Anal. Numér. (To appear.)

7. F. CHATELIN, "La méthode de Galerkin. Ordre de convergence des éléments propres, C. R. Acad. Sci. Paris Sér. A, v. 278, 1974, pp. 1213-1215.

8. J. DOUGLAS, T. DUPONT \& L. WAHLBIN, "Optimal $L_{\infty}$ error estimates for Galerkin approximations to solutions of two-point boundary value problems," Math. Comp., v. 29, 1975, pp. $475-483$.

9. G. J. FIX, "Eigenvalue approximation by the finite element method," Advances in Math., v. 10,1973 , pp. 300-316.

10. L. HERRMANN, "Finite element bending analysis for plates," J. Engrg. Mech. Div. ASCE, v. 93, 1967, pp. 13-26.

11. W. G. KOLATA, "Approximation in variationally posed eigenvalue problems," Numer. Math., v. 29, 1978, pp. 159-171.

12. S. NEMAT-NASSER, "General variational methods for elastic waves in composites," $J$. Elasticity, v. 2, 1972, pp. 73-90.

13. S. NEMAT-NASSER, "Harmonic waves in layered composites," J. Apph Mech., v. 39, 1972, pp. 850-852.

14. S. NEMAT-NASSER, "General variational principles in nonlinear and linear elasticity with applications," Mechanics Today, vol. 1, Pergamon Press, New York, 1974, pp. 214-261.

15. S. NEMAT-NASSER \& F. FU, "Harmonic waves in layered composites: bounds on frequencies," J. Appl. Mech., v. 41, 1974, pp. 288-290.

16. S. NEMAT-NASSER \& S. MINOGAWA, "Harmonic waves in layered composites: comparison among several schemes." (Preprint.)

17. J. A. NITSCHE, private communication.

18. J. S. NITSCHE \& A. H. SCHATZ, "On local approximation properties of $L_{2}$-projections on spline subspaces," Applicable Anal., v. 2, 1972, pp. 161-168.

19. J. A. NITSCHE \& A. H. SCHATZ, "Interior estimates for Ritz-Galerkin methods," Math. Comp., v. 28, 1974, pp. 937-958.

20. D. NORRIE \& G. de VRIES, Finite Element Bibliography, Plenum Press, New York, 1976.

21. J. T. ODEN \& J. N. REDDY, "Mathematical theory of mixed finite element approximations," Quart. Appl. Math., v. 33, 1975, pp. 255-280.

22. J. E. OSBORN, "Spectral approximation for compact operators," Math. Comp., v. 29, 1975 , pp. $712-725$.

23. R. STRICHARTZ, "Multipliers on fractional Sobolev spaces," J. Math. Mech., v. 16, 1967, pp. $1031-1060$.

24. P. A. RAVIART \& J. M. THOMAS, "Primal hybrid finite element methods for 2nd order elliptic equations," Math. Comp., v. 31, 1977, pp. 391-413.

25. M. I. VISHIK, "The Sobolev-Slobodetski spaces of changing order with weighted norms and applications to elliptic boundary value problems of mixed type," Partial Differential Equations, "Nauka", Moscow, 1970, pp. 71-76.

26. M. I. VISHIK \& G. I. ESKIN, "Equations in convolutions in a bounded region," Russian Math. Surveys, v. 20, 1965, pp. 85-151.

27. M. I. VISHIK \& G. I. ESKIN, "Elliptic equations in convolution in a bounded domain and their applications," Russian Math. Surveys, v. 22, 1967, pp. 13-75.

28. M. I. VISHIK \& G. I. ESKIN, "Sobolev-Slobodecky spaces of variable order with weighted norms and their applications to mixed boundary value problems," Sibirsk. Mat. Ž., v. 9, 1968, pp. 973-997.

29. A. UNTERBERGER, "Sobolev spaces of variable order and problems of convexity for partial differential operators with constant coefficients," Colloq. Internat. CNRS sur les Équations aux Dérivées Partielles Linéaires (University Paris-Sud, Orsay, 1972), pp. 325-341.

30. A. UNTERBERGER, "Espaces de Sobolev d'ordre variable et applications," Séminaire, Goulaouic-Schwartz 1970-1971, Equations aux Dérivées Partielles et Analyse Fonctionnelle, Exposé no. 5, Centre de Math., École Polyt'ech., Paris, 1971. 